\title{
1 Genetic determinants facilitating the evolution of resistance to carbapenem antibiotics
}

2 Peijun $\mathrm{Ma}^{1,2,3}$, Lorrie L. He ${ }^{1}$, Alejandro Pironti ${ }^{1}$, Hannah H. Laibinis ${ }^{1}$, Christoph M. Ernst ${ }^{1,2,3}$,

3 Abigail L. Manson ${ }^{1}$, Roby P. Bhattacharyya ${ }^{1,4}$, Ashlee M. Earl ${ }^{1}$, Jonathan Livny ${ }^{1}$, Deborah T.

4 Hung ${ }^{1,2,3, *}$

5

$6{ }^{1}$ The Broad Institute of MIT and Harvard, Cambridge, United States

7 2Department of Molecular Biology and Center for Computational and Integrative Biology,

8 Massachusetts General Hospital, Boston, United States

$9 \quad{ }^{3}$ Department of Genetics, Harvard Medical School, Boston, United States

$10{ }^{4}$ Division of Infectious Diseases, Massachusetts General Hospital, Boston, United States

11

12

13 * Address correspondence to Deborah T. Hung: hung@molbio.mgh.harvard.edu

14

15

16

17

18

19

20

21 


\section{Abstract}

In this era of rising antibiotic resistance, in contrast to our increasing understanding of

24 mechanisms that cause resistance, our understanding of mechanisms that influence the

25 propensity to evolve resistance remains limited. Here, we identified genetic factors that facilitate

26 the evolution of resistance to carbapenems, the antibiotic of "last resort," in Klebsiella

27 pneumoniae, the major carbapenem resistant species. In clinical isolates, we found that high-

28 level transposon insertional mutagenesis plays an important role in contributing to high-level

29 resistance frequencies in several major and emerging carbapenem-resistant lineages. A

30 broader spectrum of resistance-conferring mutations for select carbapenems such as

31 ertapenem also enables higher resistance frequencies and importantly, creates stepping-stones

32 to achieve high-level resistance to all carbapenems. These mutational mechanisms can

33 contribute to the evolution of resistance, in conjunction with the loss of systems that restrict

34 horizontal resistance gene uptake, such as the CRISPR-Cas system. Given the need for

35 greater antibiotic stewardship, these findings argue that in addition to considering the current

36 efficacy of an antibiotic for a clinical isolate in antibiotic selection, considerations of future

37 efficacy are also important. The genetic background of a clinical isolate and the exact antibiotic

38 identity can and should also be considered as it is a determinant of a strain's propensity to

39 become resistant. Together, these findings thus provide a molecular framework for

40 understanding acquisition of carbapenem resistance in $K$. pneumoniae with important

41 implications for diagnosing and treating this important class of pathogens.

\section{Introduction}

Antibiotic resistance is one of the most urgent threats to public health. Resistance has

44 emerged to almost all clinically used antibiotics and in nearly all bacterial pathogen species.

45 Numerous studies have focused on identifying and characterizing resistance mechanisms; 
meanwhile, our understanding of mechanisms that facilitate the evolution of resistance in clinical

47 isolates is less well understood ${ }^{1}$. As such, antibiotic efficacy as reflected in minimum inhibitory concentrations (MICs) remains almost the sole criterion to guide clinical antibiotic choice. However, more sophisticated antibiotic stewardship that considers the frequency of the evolution of resistance in antibiotic selection would help to preserve the existing arsenal of antibiotics. Such stewardship would need to be informed by an increased understanding of the

52 mechanisms that may affect the evolution of resistance including microbial intrinsic factors such

53 as the genetic background of an isolate and extrinsic factors such as the antibiotic choice.

Bacteria acquire antimicrobial resistance through horizontal gene transfer (HGT) or mutation, processes that can be influenced by intrinsic microbial genetic factors, such as phage defense systems and error prone polymerases, respectively ${ }^{2,3}$. While HGT involves the

57 acquisition of new resistance genes, mutation of existing genes can occur by acquisition of single nucleotide polymorphisms, insertions, deletions, recombination, or transposition events. At the same time, microbe extrinsic factors such as the antibiotic identity can also affect the evolution of resistance, as they vary in their ability to induce mutagenesis ${ }^{4}$, have different barriers to resistance ${ }^{5}$, and vary in their spectrum of possible resistance conferring mutations.

63 infections resistant to almost all antibiotics including extended spectrum $\beta$-lactam antibiotics ${ }^{6,7}$.

64 Carbapenem resistance thus typically emerges in bacteria that already carry extended spectrum

$65 \beta$-lactamases (ESBLs) and/or other $\beta$-lactamases ${ }^{8-10}$. Carbapenem resistance is most often

66 mediated by the production of carbapenemases. In the absence of carbapenemases however,

67 resistance can be achieved through the acquisition of a combination of porin mutations to

68 impede drug entry and/or significant increases in $\beta$-lactamase expression ${ }^{8-11}$. Therefore, the

69 evolution of carbapenem resistance often involves complex mechanisms of HGT and mutation 70 acquisition. 
The Gram-negative pathogen Klebsiella pneumoniae is one of the most prevalent

72 carbapenem resistant Gram-negative species ${ }^{12,13}$. Within this species, carbapenem-resistance

73 occurs predominantly in a few clonal groups (CG), such as CG258, CG15, and CG208,13-18.

74 While clonal spread plays a role in the dissemination of carbapenem resistance ${ }^{8,15}$, the

75 emergence of new highly resistant lineages ${ }^{19-24}$ and the independent acquisition of carbapenem

76 resistance by distinct CG258 strains ${ }^{14,20,25,26}$ suggest that ongoing evolution of carbapenem

77 resistance also plays an important role. These observations suggest that the underlying genetic

78 background of CG258 and other emerging lineages may contribute to a higher propensity for

79 resistance acquisition. Recently, many bioinformatic studies have reported that a major phage

80 defense system, the CRISPR-Cas system, is absent in Sequence Type ST258 and ST11

81 strains, two major lineages of CG258 ${ }^{27-29}$. As one of the earliest lineages causing outbreaks of

82 carbapenem resistance, ST258 K. pneumoniae isolates are responsible for the global spread of

83 K. pneumoniae carbapenemases $(\mathrm{KPC})^{8,15}$. Therefore, it has been suggested that the lack of

84 CRISPR-Cas systems could be one of the genetic factors contributing to the high rates of

85 carbapenem resistance in this group. However, the more recently emerging lineages, such as

86 ST15 and ST307 do contain such systems and so carbapenem resistance more generally

87 cannot be explained so simply.

Meanwhile, antibiotic identity may also affect the frequency of evolving resistance.

89 Currently, four different carbapenems are available in an intravenous formulation ${ }^{6}$ : imipenem,

90 meropenem, ertapenem, and doripenem. In addition, faropenem, a related oral antibiotic in the

91 penem class, is available but only outside of the U.S. ${ }^{30}$. Although the five drugs share similar

92 structures and mechanisms of action, differences in their pharmacokinetics (ertapenem can be

93 administered once a day while the other carbapenems require administration 3-4 times per day),

94 stability against $\beta$-lactamase hydrolysis, and penicillin-binding protein target preference ${ }^{7,31-33}$

95 may influence the evolution of resistance differently. For example, previous studies have shown 
96 that compared to other carbapenems, ertapenem is more susceptible to hydrolysis by some $\beta$ -

97 lactamases and its cell entry is more impeded by the loss of porins ${ }^{34,35}$, raising the possibility

98 that a broader spectrum of mutations on $\beta$-lactamase or porin genes may selectively affect

99 ertapenem but not the other carbapenems.

In this study, to understand how bacterial genetic background and different

101 carbapenems affect the rates of resistance evolution, we compared mutation frequencies

102 (previously defined as the frequency of independent resistant mutants emerging in a given

103 population ${ }^{36}$ ) of carbapenem-susceptible K. pneumoniae clinical isolates from ten lineages and

104 found that isolates from the dominant and emerging carbapenem-resistant lineages had higher

105 mutation frequencies leading to carbapenem resistance than other lineages. We demonstrated

106 that the higher mutation frequencies are caused by high-level transposon insertional

107 mutagenesis, a process leading to resistance gene duplication and reversible porin disruption.

108 We also showed experimentally that one of the major phage defense systems, CRISPR-Cas

109 systems, indeed can play a role in restricting resistance gene acquisition when corresponding

110 spacers sequences are present. Furthermore, we found that a broad spectrum of resistance-

111 conferring mutations for selected carbapenems such as ertapenem contributed to increased

112 resistance rates; importantly, these mutations selected from ertapenem exposure could serve

113 as stepping stones to high-level resistance to all carbapenems. Taken together, this work

114 identified multiple factors that facilitate the evolution to carbapenem resistance in $K$.

115 pneumoniae clinical isolates, and demonstrates that the evolution of antibiotic resistance can be

116 a complex process with important implications for antibiotic selection tailored to the genetic

117 background of clinical isolates.

\section{Results}


The evolution of carbapenem resistance was affected by genetic background of the isolates

We analyzed genomes of 267 previously sequenced K. pneumoniae clinical isolates ${ }^{8}$

122 and selected carbapenem-susceptible isolates from ten lineages (Fig. 1, Supplementary Table

123 1\&2). We chose isolates from (1) the predominant carbapenem-resistant lineage that has

124 caused many outbreaks since 2000 (UCI38 (ST258)), (2) the dominant ESBL-producing lineage

125 that is becoming increasingly carbapenem-resistant (MGH222 (ST15)), (3) newly emerging

126 carbapenem-resistant lineages (UCICRE126 (ST147), MGH66 (ST29), BIDMC41 (ST37),

127 MGH74 (ST76), MGH158 (ST152), UCI64 (ST17)), and (4) lineages that have not caused

128 carbapenem-resistant clonal outbreaks (UCI34 (ST34) and MGH21 (ST111)). We measured

129 mutation frequencies of these isolates under ertapenem (Fig. 2a) or rifampicin treatment (Fig.

$1302 \mathrm{2b})$, using a modified Luria-Delbrück system in which low numbers of bacterial cells were

131 seeded into each well of 384-well plates, thus making the emergence of two independent

132 mutants in the same well extremely unlikely ${ }^{37}$ (Supplementary Fig. 1). This format requires that

133 all resistance occurs through mutation acquisition and not HGT. (We define resistance as at

134 least a 2-fold increase in the MIC for the mutant relative to the MIC against the original

135 susceptible parent strain, and not relative to the clinically defined MIC breakpoints of the

136 antibiotic. Therefore, resistant mutants selected from our experiments do not necessarily have

137 MICs that are greater than the clinical breakpoints.) We found that except for MGH66 (ST29), all

138 isolates showed similar levels of mutation frequencies to rifampicin (Fig. 2b), whereas a wide

139 range of mutation frequencies to ertapenem were observed (Fig. 2a). In particular, some strains

140 had much higher mutation frequencies to ertapenem than to rifampicin. Since resistance to

141 rifampicin is acquired through point mutation resulting from errors during DNA replication ${ }^{38,39}$,

142 these results suggest that other genetic mechanisms help to determine the mutation frequency

143 to ertapenem. 
Among all strains tested, UCI38 (ST258) had the highest mutation frequency to ertapenem. It carries an ESBL gene blasHV-12 on the plasmid pESBL (Fig. 2c), raising the possibility that ESBL activity could contribute to high-level mutation frequencies. To test this hypothesis, we transformed pSHV (Fig. 2d), a multi-copy laboratory plasmid containing blasHV-12, amplified from pESBL, into three isolates lacking an ESBL gene and with baseline low-level mutation frequencies to ertapenem, including UCI64 (ST17), UCI34 (ST34), and MGH21 (ST111). However, introduction of blasHV-12 did not change the mutation frequencies of these strains for ertapenem (Fig. 2e), even though the expression of blasHV-12 was higher in strains

152 transformed with pSHV than in UCI38 which naturally carries blasHV-12 (Supplementary Fig. 2).

153 This ruled out the simple presence of the ESBL gene alone as the reason for the differing 154 mutation frequencies. confer high-level mutation frequencies to ertapenem. However, when we attempted to transform pESBL into the same three strains with low-level mutation frequencies to ertapenem, none of them could take up pESBL. In contrast, an ST258 strain BWH41 (the only ST258 isolate lacking an ESBL gene in our collection) and a laboratory strain of E. coli, $10 \beta$, could take up pESBL (Fig. 2f). Meanwhile, all strains successfully took up pSHV with similar efficiencies, suggesting that $\mathrm{pESBL}$ was uniquely restricted in particular strains. via HGT while other genetic factors contribute to high mutation frequencies we analyzed the genomic sequences of the collection of $267 \mathrm{~K}$. pneumoniae isolates for the presence of two major phage-defense systems, the CRISPR-Cas systems and Restriction167 modification systems (Supplementary Table 3), which function to exclude foreign DNA. We 
found that of the 3 strains which could not take up pESBL, MGH21 (ST111) and UCI34 (ST34) have type I CRISPR-Cas systems, while UCI64 (ST17) has no CRISPR-Cas system but carries

170 Type I R-M systems. In contrast, among 80 strains of the ST258 lineage, we found no CRISPR-

171 Cas systems and most strains carry Type III R-M system (Fig. 1 \& Supplementary Table 3).

172 When we broadened our analysis to include the genomic sequences of $2453 \mathrm{~K}$. pneumoniae

173 strains available in the NCBI database, including 550 ST258 strains, we found that no ST258

174 strains contain a CRISPR-cas system (Supplementary table 4), confirming that the lack of

175 CRISPR-Cas system is a genetic feature of the ST258 lineage. This finding is consistent with

176 other bioinformatic studies which have tried to link the absence of CRISPR systems in ST258

177 strains to carbapenem resistance ${ }^{27-29}$. However, there is no clear association between the

178 absence of CRISPR and the more recently emerging carbapenem-resistant lineages (Fig. 1 and

179 Supplementary Table 3\&4).

To understand the ability of MGH21 (ST111) to restrict pESBL uptake, a strain which encodes a Type I-E CRISPR-Cas system but no R-M systems, we first confirmed by RNA sequencing (RNA-seq) that indeed the CRISPR-Cas system was expressed in MGH21

183 (Supplementary Fig. 3). We then compared the sequence of pESBL with MGH21's CRISPR-

184 Cas system and found that MGH21 has a spacer (spacer 11) (Fig. 3a \& Supplementary Table 5) targeting a gene encoding a DNA-methyltransferase (DNMT) ${ }^{40}$ in pESBL (Fig. 2c); by searching a curated plasmid database ${ }^{41}$, we found that this spacer additionally aligns with sequences

187 found in an additional 94 other plasmids carrying antibiotic resistance genes, including 62 multi188 drug resistance plasmids (plasmids carrying resistance genes to more than one class of 189 antibiotics) and 21 plasmids carrying carbapenemase genes (Supplementary Table 6 and 7). In 190 addition, spacer24 (Fig. 3a \& Supplementary Table 4) aligned to a conserved hypothetical gene 191 that was also found in UCI38 as well as 66 additional plasmids carrying antibiotic resistance 192 genes, including 44 multi-drug resistant plasmids and 12 plasmids carrying carbapenemase 
193 genes (Supplementary Table 6 and 8). Collectively, these results pointed to the potential role of

194 the CRISPR-Cas system in excluding the uptake of resistance carrying plasmids such as

195 pESBL. Indeed, after depleting the CRISPR-Cas operon (MGH21 $\Delta$ cas; the CRISPR-Cas

196 system along with two adjacent hypothetical genes was deleted), pESBL could now be

197 successfully transformed, whereas episomal complementation of the CRISPR-Cas system back

198 into $\mathrm{MGH} 21 \Delta$ cas again restricted pESBL transformation (Fig. 3b).

Unsurprisingly, the absence of CRISPR-Cas system increased rates at which resistance

by HGT could be acquired but did not change mutation frequencies of MGH21 (Fig. 3d). In

201

contrast, introduction of pESBL into $\mathrm{MGH} 21 \Delta$ cas increased the frequency with which resistance

202 to ertapenem emerged in our modified Luria-Delbruck system where HGT cannot occur; the

203 frequency for MGH21 $\Delta$ cas (pESBL) was 30 times higher than for the parent MGH21,

204 MGH21 $\Delta$ cas, or MGH21 carrying pSHV (Fig.3d). As introduction of the ESBL gene alone in

205 pSHV does not change resistance frequencies, this elevation suggests that factors on pESBL

206 other than the ESBL gene contributed to the high mutation frequencies. Further, while

$207 \mathrm{MGH} 21 \Delta \operatorname{cas}(\mathrm{pESBL})$ had elevated ertapenem mutation frequencies relative to MGH21, its

208 frequency was still 10-20 times lower than that of UCI38 itself, from which pESBL was isolated

209 (Fig. 3d), suggesting that differences between the genetic backgrounds of MGH21 and UCI38,

210 irrespective of pESBL, play additional roles in high frequency mutation acquisition.

211 Transposon insertional mutagenesis caused frequent and reversible inactivation of porin

212 genes leading to ertapenem resistance

To gain insight into other genetic factors that may cause the different levels of mutation

214 frequencies to ertapenem between $\mathrm{UCl} 38$ and MGH21, we analyzed whole genome sequencing

215 (WGS) data of laboratory-derived resistant mutants to identify the specific genetic events

216 leading to ertapenem resistance. We compared six ertapenem resistant mutants derived from 
217 UCI38 (ST258), five mutants derived from MGH21 (ST111), and five mutants derived from

$218 \mathrm{MGH} 21 \Delta \operatorname{cas}(\mathrm{pESBL})$ (Fig. 4a), We found that the two strains carrying pESBL favored

219 transposition events as a mechanism to attain resistance while the strain lacking $\mathrm{pESBL}$,

220 MGH21, developed resistance only through SNP acquisition. All six resistant mutants derived

221 from UCl38 were due to duplication of the transposon on pESBL in which the blasHV-12 is

222 embedded (Fig. 2c) and/or disruption of ompK36, one of the major porin genes of $K$.

223 pneumoniae that facilitates carbapenem cell entry, by insertion sequences (ISs, small

224 transposons that only carry the transposase genes). (Although the other porin OmpK35 also

225 facilitates cell entry for carbapenems, we found no resistant mutants carrying mutations in

226 ompK35, probably due to the low expression levels of ompK35 in the growth condition used ${ }^{42}$

227 (Supplementary Fig. 4) or pre-existing mutations already disrupting ompK35 $5^{15}$ in some strains.)

228 Similarly, for MGH21 1 cas(pESBL), four mutants stemmed from the same transposon

229 duplication of blasHV-12 on pESBL, while the fifth mutant resulted from the acquisition of a SNP in

230 ompK36. In contrast, all resistant mutants derived from MGH21 resulted from the acquisition of

231 SNPs or short deletions/insertions, mostly in porin genes and outer membrane protein genes.

$232 \mathrm{pESBL}$ thus increased mutation frequencies relative to $\mathrm{pSHV}$ because blasHV-12 on pESBL lies

233 within a transposon that can be easily duplicated to elevate ESBL expression and thus MIC

234 (Fig. 3d). In contrast, while carrying pSHV intrinsically conferred a higher baseline MIC because

235 of its higher bla $\mathrm{SHV}_{-12}$ expression level (Supplementary Fig. 2), it could not duplicate blasHV-12 to

236 further evolve increased MIC, thus explaining its unchanged mutation frequencies relative to the

237 parent MGH21 (Fig. 1e).

Comparing the two strains that carry pESBL, we noted that UCI38 was able to disrupt

239 ompK36 through transposon insertion while MGH21 $\Delta$ cas (pESBL) only did so through SNP

240 acquisition. We hypothesized that the higher likelihood of a disrupting transposition event rather

241 than the acquisition of a disrupting SNP might explain the higher mutation frequencies of UCI38 
242 and other strains with relatively high-level mutation frequencies to ertapenem (Fig. 2a). Indeed,

243 when we used the modified Luria-Delbrück system to isolate and characterize 50 to 100

244 ertapenem-resistant mutants from each of these ten isolates (Supplementary Fig. 5;

245 Supplementary Table 9), we found that transposon insertions in ompK36 accounted for $60-90 \%$

246 of resistant mutants derived from strains with high-level mutation frequencies to ertapenem,

247 while only $0-10 \%$ of mutants resulted from transposon insertion in ompK36 in strains with

248 relatively lower mutation frequencies to ertapenem (Fig. 4b). Of note, no one specific IS element

249 accounted for the high transposition rates, as ISs from four different families (IS4, IS5, IS91,

250 IS1) were involved in the inactivation of ompK36 (Fig.4c and Supplementary Table 10). There

251 was also no correlation between the number of ISs and the activity-level of transposon

252 insertional mutagenesis (Supplementary Fig.6). Nevertheless, these results demonstrate that a

253 higher propensity for transposon insertional mutagenesis in some genetic backgrounds was an

254 important contributor to the more facile evolution of ertapenem resistance in some strains, with

255 such events occurring at nearly ten times higher frequency than SNP acquisition.

257 insertions can be reversible ${ }^{43}$. Since porin disruption is known to come at a fitness cost in the

258 absence of antibiotic selective pressure ${ }^{44,45}$, the mechanism of transposon disruption of ompK36

259 to achieve antibiotic resistance in UCI38 afforded a potentially facile path, i.e., reversion, to

260 recover from this fitness cost when selective pressure is removed. Indeed, this reversion was

261 observed when we cultured Mut41 (Fig. 4c), a mutant of UCI38 carrying an IS1 insertion in the

262 promoter region of ompK36, without antibiotics (Fig.4d). 99\% of the population reverted to the

263 wild-type ompK36 gene by 100 generations, thereby restoring both the expression of ompK36

264 and the fitness of the strain relative to the parent mutant Mut41 (Fig. 4e and 4f). We observed

265 the same phenomenon in mutants derived from three other strains (Supplementary Fig. 7)

266 demonstrating the high versatility of this resistance mechanism. A high propensity for 
267 transposon insertional mutagenesis resulting in porin inactivation provides a fitness advantage

268 in the presence of antibiotic, while preserving a path to restoration of fitness in the absence of 269 antibiotics.

270 Spectrum of genetic mutations conferring resistance to ertapenem is broader than to 271 meropenem

273 evolves. We measured mutation frequencies in response to treatment with four carbapenems

274 and faropenem in three representative carbapenem-susceptible K. pneumoniae clinical isolates:

275 UCI38 (an ST258 strain carrying one chromosomal ESBL blasHV-12 and a second episomal

276 blasHV-12 copy), MGH21 (an ST111 strain with a single copy of the non-ESBL blasHV-11 on the

277 chromosome), and MGH32 (an ST111 strain with no $\beta$-lactamase genes because the single

278 native, chromosomal blasHv-1 is inactivated) (Fig. 5a and Supplementary Table 1). The lowest

279 mutation frequencies resulted from meropenem treatment while relatively higher frequencies

280 resulted from ertapenem and faropenem. In the case of MGH32, which carries no $\beta$-lactamase

281 gene, we did not isolate resistant mutants to any of the carbapenems including ertapenem, but

282 isolated resistant mutants to faropenem (Fig. 5a), indicating that $\beta$-lactamase genes may be

283 necessary for the evolution of resistance to carbapenems but not to faropenem. To confirm that

284 our observation was not limited to these three strains, we measured mutation frequencies of an

285 additional three isolates under separate treatment of these five antibiotics, and similar patterns

286 were observed, suggesting that the influence of carbapenem identity is independent of the

287 genetic background of strains (Supplementary Fig. 8).

289 (Supplementary Fig. 9a), and bacteria were treated with concentrations of antibiotic normalized 290 to their MICs for each drug, the different mutation frequencies were not explained by differences 
291 in antibiotic exposure. We also ruled out the possibility that ertapenem could induce more

292 mutagenesis than meropenem, a phenomenon that has been described for some $\beta$-lactams ${ }^{46}$,

293 by measuring the mutation frequencies to rifampin after pre-treatment with sub-MIC

294 concentrations of ertapenem, meropenem, or ciprofloxacin (a fluoroquinolone antibiotic known

295 to induce mutagenesis ${ }^{4}$ ) as a positive control. While both carbapenems increased rifampin

296 mutation frequencies compared with untreated controls, each did so equivalently, and less than

297 ciprofloxacin (Supplementary Fig. 9b).

We then turned to the possibility that ertapenem's higher mutation frequency could be

299 due to a greater spectrum of resistance-conferring mutations than for meropenem. We isolated

300 and characterized 90 mutants, derived from UCI38 or MGH21, that were selected from our

301 modified Luria-Delbrück system with confirmed shifts in the corresponding MICs of ertapenem

302 and meropenem (Table 1 and Supplementary Table 10). Sixty-three mutants had increases in

303 the MICs, relative to their corresponding ancestor strains, of both ertapenem (2-256 folds

304 increases) and meropenem, albeit with relatively lower levels of meropenem resistance (2-16

305 folds increases). We did not isolate any mutants that are highly resistant (MIC $>4 \mu \mathrm{g} / \mathrm{mL})$ to

306 meropenem. Meanwhile, 27 mutants only had corresponding increases in the MICs of

307 ertapenem, and not meropenem (Supplementary Table 11). No mutants had an increased MIC

308 of meropenem but not ertapenem.

We analyzed WGS data from ten representative mutants, five that had MIC shifts to both

310 ertapenem and meropenem, and five that had MIC shifts only to ertapenem (Table1), and

311 validated all identified resistance-conferring mutations by complementation (Supplementary

312 Table 12). Six of the mutants contained either transposon insertions or SNPs in ompK36 or

313 duplication of blasHV-12. Interestingly, four mutants carried novel mutations, including mutations

314 in wzc (capsule synthesis), ompA (porin), resA (anti-sigma $\mathrm{E}$ factor), and the promoter region of

315 bamD (outer membrane protein assembly factor), with the first three resulting in selective 
ertapenem resistance. These results show that indeed ertapenem had a wider allowable

317 spectrum of resistance-conferring mutations than meropenem, which yielded a higher mutation

318 frequency.

\section{Pre-selection with ertapenem increased the likelihood of evolving resistance to} meropenem both by spontaneous mutation and HGT

While many ertapenem resistant mutants do not display resistance to meropenem, we

322 found that acquisition of such mutations, while not impacting the immediate efficacy of

323 meropenem as reflected in the MIC, impacted its future efficacy by increasing the frequency at

324 which resistance to meropenem emerges. The mutation frequencies of an ertapenem-restricted

325 resistant strain (Mut34, which carries a duplication of blasнv on pESBL (Table 1)) were more

326 than 100 times greater than the frequency of its corresponding parental strain $\mathrm{UCI} 38$ under

327 identical meropenem treatment (Fig. 5b). WGS of the meropenem resistant mutants revealed

328 that the majority of the mutants derived from Mut34 had acquired new mutations in the porin

329 gene ompK36 (i.e., Mut186, Fig. 5c, d), to accompany the previously acquired blasHV-12

330 duplication. These results demonstrate that the previously acquired mutation in Mut34 that

331 confers ertapenem resistance alone, could serve as a stepping-stone to the subsequent

332 acquisition of a porin disrupting mutation to yield meropenem resistance.

Of note, de novo mutation acquisition, even in this stepping-stone fashion, resulted in

334 only low to moderate levels of meropenem resistance (4 - 32 folds increase in MIC from the

335 ancestor strains). With the hypothesis that HGT of carbapenemases or additional ESBL genes

336 may be required to evolve truly high-level meropenem resistance, we examined the impact of

337 the ertapenem-limited resistance mutations on the ability to horizontally acquire resistance

338 genes. Indeed, in the presence of meropenem, higher rates of uptake of a clinical plasmid

339 carrying the carbapenemase gene blakPC-2 were observed for both Mut34 and Mut186 than the 
ertapenem sensitive parental strain UCI38 (Fig. 5e); rather than a direct mechanistic impact, this

341 finding is likely due to longer survival times of these mutants in the presence of meropenem

342 compared to the parental strain affording them a greater opportunity to pick up the plasmid, as

343 the conjugation frequencies are the same in the absence of meropenem (Supplementary Fig.

344 10). A faropenem-limited resistant mutant, Mut101, like Mut34 for ertapenem, also showed

345 elevated mutation frequencies and conjugation efficiencies in the presence of meropenem

346 compared to its parental strain (Supplementary Table 1 and Supplementary Fig. 11). Together

347 these results suggest that ertapenem and faropenem not only elicit more frequent resistance

348 themselves, but they also select for mutations that can increase the rates at which bacteria

349 acquire high-level meropenem resistance.

350

351

352

353

354

355

356

357

\section{Discussion}

In this study, we identified genetic factors that facilitate the evolution of carbapenem resistance in K. pneumoniae clinical isolates (Supplementary Fig. 12), one of the most alarming antibiotic-resistant pathogens that have emerged due to our limited arsenal against such organisms. We find that high-level transposon insertional mutagenesis and the mutational spectrum for each carbapenem play important roles in increased mutation frequencies. These mutational mechanisms can work in conjunction with loss of systems that restrict horizontal resistance gene uptake, i.e., the CRISPR-Cas systems, to facilitate the evolution of resistance.

We found that isolates of major and emerging carbapenem-resistant lineages indeed have high-level mutation frequencies to carbapenem antibiotics compared to lineages which have not been linked to carbapenem resistance; this is due to high-level transposon insertional mutagenesis in lineages associated with carbapenem resistance. This highlights the notion that the emergence of predominant resistant lineages did not occur through random events and provide genetic markers that signal isolates with high risk of developing resistance. Importantly, 
364 this mechanism of acquiring resistance could serve an evolutionary advantage as the disruption

365 of porins by transposons can revert (Fig. 4d), thereby enabling strains to rapidly adapt to

366 fluctuating environments and optimizing their survival in the presence and absence of antibiotic

367 exposure. The fact that many of the more recently emerging lineages, such as ST15 and

368 ST307, have evolved resistance by a combination of ESBLs and porin truncations may

369 potentially point to the relevance of such mutagenic mechanisms. More generally, transposon-

370 mediated gene duplication has been reported to contribute to heteroresistance in many different

371 bacterial species and antibiotic classes ${ }^{47,48}$. This study thus provides further evidence that

372 mutational events mediated by transposons play a critical role in the evolution of antibiotic

373 resistance in parallel with HGT.

Bioinformatic studies have previously suggested a potential relationship between the

375 absence of CRISPR-Cas systems and carbapenem resistance in the ST258 lineage ${ }^{27-29}$.

376 However, as the more recent resistant lineages to emerge still retain CRISPR-Cas systems, the

377 absence of such systems cannot fully explain the emergence of resistance. Here we

378 demonstrated that they indeed can play a role in restricting the uptake of resistance plasmids, if

379 accompanied by appropriate spacers (Fig. 3). Importantly, bioinformatic analysis of spacer

380 sequences, and not simply the presence or absence of a CRISPR-Cas system alone, is needed

381 to understand the functional role of such systems in resistance gene exclusion in the recently

382 resistant lineages.

The mutational spectrum that confers resistance to each carbapenem also affects

384 evolution frequencies. Currently in practice, several factors affect the choice of a specific

385 carbapenem or faropenem in treating a patient, including its availability, spectrum of activity,

386 dosing schedule, route of administration, and cost. Ertapenem is sometimes favored for the

387 convenience of its once-daily dosing, whereas the other three carbapenems all require 3-4

388 doses per day. However, ertapenem and faropenem lack activity against Pseudomonas 
aeruginosa, thus limiting their use in some infections ${ }^{7,49}$. Besides these factors, mutation frequencies associated with these antibiotics have not been taken into consideration in antibiotic prescription. In this study, we show that a higher resistance frequency is associated with ertapenem and faropenem due to the broader spectrum of resistance-conferring mutations than is allowed for other carbapenems such as meropenem. Importantly, these mutations can serve as stepping-stones to facilitate the evolution of high-level resistance to all carbapenems. As ertapenem or faropenem are often favored for the convenience of its once-daily dosing or oral bioavailability, respectively, these results highlight the non-equivalence of antibiotics even within

397 the same class of antibiotics with respect to the propensity to evolve resistance. It might suggest

398 that the use of carbapenems with a higher barrier to resistance should be favored to prevent the evolution of carbapenem resistance. MIC as an indicator of susceptibility. However, this work shows that treating strains with similar

402 MICs with the same antibiotic could have different outcomes with regards to the emergence of 403 resistance. Isolates with diverse genetic backgrounds can have very different mutational 404 frequencies, despite having the same MIC (Supplementary Table 1). Clearly, some genetic mutations pre-selected from ertapenem or faropenem treatment are not sufficient to change MICs of meropenem, but they can significantly increase the likelihood of evolving resistance to 407 meropenem. the discovery of new antibiotics, parallel efforts are needed to guide more judicious use of our

410 current available antibiotics to minimize the emergence of resistance. This work suggests that

411 strategies should not only consider current efficacy, but also consider both the genetic

412 backgrounds of strains and antibiotic choice as they impact the potential for erosion of future

413 efficacy. More generally, this work demonstrates that investigating evolutionary drivers of 
414 antibiotic resistance can reveal the root causes of resistance evolution, thereby providing a

415 framework to improve current clinical diagnosis and antibiotic selection.

\section{Methods}

\section{Modified Luria-Delbrück experiment}

418 The robotic, modified Luria-Delbrück system was adapted from a system that was previously

419 described by Gomez et al. ${ }^{37}$. Exponential growth phase bacterial cultures were diluted to

420 roughly 100 cells per $50 \mu \mathrm{l}(2000$ cells $/ \mathrm{ml})$ in MHB medium. Then the diluted culture was

421 transferred to three to six 384-well microplates (Falcon, cat\#. 353962) using Bravo liquid

422 handling platform (Agilent), and each well of these 384 -well plates contained $50 \mu \mathrm{l}$ of the

423 culture. The plates were sealed using BioExcell Film for Tissue Culture (Worldwide life science,

424 cat. \# 41061023) and placed in humidified containers at $37^{\circ} \mathrm{C}$. After incubating for three hours,

$42510 \mu \mathrm{l}$ cultures were taken from three randomly selected wells and diluted for plating on LB agar

426 plates to quantify cell numbers. Then antibiotics at specified concentrations were added to the

427 wells using Bravo liquid handling platform at specified concentrations. After adding antibiotics,

428 cultures were incubated in humidified containers at $37^{\circ} \mathrm{C}$ overnight. The second day morning,

$429 \mathrm{OD}_{600}$ was read using SpectroMax plate reader (Molecular Device) and mutation frequency was

430 calculated using the following equation: $1-\sqrt[c]{\left(\frac{w_{n}}{w_{t}}\right)}$, where $c$ is the number of cells per well at the

431 time of adding antibiotics, $w_{n}$ is the number of negative wells, and $w_{t}$ is the total number of

432 wells. Mutants from each plate were sub-cultured in MHB supplemented with the same

433 antibiotics at the same concentrations used for the selection, and saved in $25 \%$ glycerol stocks

434 for future analysis. Mutants that did not grow up in the sub-culturing were excluded from the

435 calculation of mutation frequencies. Each experiment was repeated three times. 
436 To measure mutation frequencies with rifampin after carbapenem treatment, exponential growth

437 phase cultures of $\mathrm{UCI} 38\left(\mathrm{OD}_{600} \sim 0.2\right)$ were diluted 100 times with MHB medium, then the

438 diluted cultures were split into four identical cultures. Cell numbers were quantified by plating

439 diluted cultures on LB agar plates, and these cell numbers were used to calculated mutation

440 frequencies. Ertapenem, meropenem and ciprofloxacin were then added to three of these four

441 cultures at $0.1 \times$ MICs. The fourth culture was untreated. Immediately after adding antibiotics,

442 cultures from each condition were aliquoted into three 384-deep-well plates (VWR, cat. \#

443 82051-326) with $50 \mu \mathrm{l}$ per well using Bravo liquid handling platform. Then these 12 384-deep-

444 well plates were incubated at $37^{\circ} \mathrm{C}$ with shaking for 2 hours. After incubating for two hours, 10

$445 \mu$ cultures from each well of these 12 deep-well plates were correspondingly transferred to wells

446 of 12 384-clear-bottom microplates (Falcon, cat\#. 353962), using Bravo liquid handling platform.

447 Then $50 \mu \mathrm{l}$ MHB supplemented with rifampin at the concentration of $60 \mu \mathrm{g} / \mathrm{ml}$ was added to

448 each of these wells. The final rifampin concentration in each well was $50 \mu \mathrm{g} / \mathrm{ml}$. These plates

449 were incubated in humidified containers at $37^{\circ} \mathrm{C}$ overnight. The second day morning, $\mathrm{OD}_{600}$

450 was read using SpectroMax plate reader (Molecular Device) and mutation frequency was

451 calculated using this equation: $1-\sqrt[c_{0}]{\left(\frac{w_{n}}{w_{t}}\right)}$, where $c_{0}$ is the number of cells per well before pre-

452 treatment, $w_{n}$ is the number of negative wells, and $w_{t}$ is the total number of wells. This

453 experiment was repeated three times.

\section{Bacterial strains and plasmid construction}

455 Bacterial strains used in this study are listed in Supplementary Table 1. All strains were cultured

456 in Luria-Bertani (LB) medium or Mueller-Hinton Broth (MHB) with shaking at $37^{\circ} \mathrm{C}$ or $30^{\circ} \mathrm{C}$ as

457 specified.

458 To construct the plasmid pSHV, blasHV-12, including the 500 base pairs (bp) upstream region, 459 were PCR amplified from UCI38, respectively, using primers listed in Supplementary Table 13. 
460 Then the PCR products were ligated into vector pSmart LC Kn (Lucigen, cat.\# 40821) and

461 electroporated into E. coli competent cells $10 \beta$ (NEB, Cat.\# C3020K). Plasmids were then

462 extracted from positive clones and electroporated into K. pneumoniae cells that have been

463 made electroporation competent according to the protocol described previously ${ }^{50}$. In brief, $K$.

464 pneumoniae cells were streaked on LB agar plates and grown overnight at $37^{\circ} \mathrm{C}$. Then cells

465 were collected directly from LB plates and re-suspended in ice-cold sterilized $\mathrm{H}_{2} \mathrm{O}$, followed by

466 washing with ice-cold sterilized $\mathrm{H}_{2} \mathrm{O}$ three times. Finally, cells were re-suspended at the

467 concentration of roughly $10^{9} \mathrm{cells} / \mathrm{ml}$ for electroporation. Strains expressing blasHV-12 were

468 cultivated in medium supplemented with kanamycin at the concentration of $25 \mu \mathrm{g} / \mathrm{ml}$.

469 To generate $\mathrm{MGH} 21 \Delta$ cas, about 1000 bp upstream and downstream of the cas operon was

470 amplified from MGH21 using Q5 DNA polymerase (NEB, Cat\# M0492). Overlap extension PCR

471 was used to fuse these two pieces of DNA to generate a 2000 bp fragment which was then

472 ligated to pKOV vector ${ }^{51}$ using BamHI and Notl sites, resulting in the construct pKOV-casKO.

473 The construct was transformed to E. coli competent cells $10 \beta$ (NEB, Cat.\# C3020K) via

474 electroporation, and the positive transformants were cultured in LB medium supplemented with

475 chloramphenicol $(34 \mu \mathrm{g} / \mathrm{ml})$ at $30^{\circ} \mathrm{C}$. Plasmids were then extracted and electroporated into

476 MGH21 electro competent cells and incubated at $30{ }^{\circ} \mathrm{C}$ on LB agar plates supplemented with

477 chloramphenicol $(34 \mu \mathrm{g} / \mathrm{ml})$ overnight. The integration of the plasmid in either the upstream or

478 the downstream region of the cas operon was selected by chloramphenicol resistance and

479 screened by PCR. Following the selection, the integrants were grown in non-selective LB

480 medium for several generations and then plated on LB agar medium with $10 \%$ sucrose to

481 induce double recombination. Among the survivors of the sucrose-LB medium, the double

482 recombinants were selected by PCR screening. The deletion of the cas operon was confirmed

483 by sequencing and RT-qPCR. 
484 To restore the CRISPR-Cas system to MGH21 1 cas, cas3, including upstream 500 bp and the

485 CRISPR array II, was amplified from MGH21 and ligated into pSmart LC Kn (Lucigen, cat.\#

486 40821), generating pCas3CRISPR2. Meanwhile, the coding region of casABECD, cas1, cas2,

487 and CRISPR array I, were amplified from MGH21 and ligated into pBAD33Gm ${ }^{52}$ using KpnI and

488 Xbal cloning sites, resulting in pBAD33Gm_CasCRISPR1. A SD sequence was also added 8 bp

489 upstream of ATG codon of casA. These two constructs were separately transformed into E. coli

$49010 \beta$ (NEB, Cat.\# C3020K) via electroporation. Plasmids were extracted, mixed at 1:1 ratio, and

491 transformed into $\mathrm{MGH} 21 \Delta$ cas, generating the strain MGH21 $\Delta$ cas(pCas). The transformants

492 containing these two constructs were confirmed using PCR and Sanger sequencing. Similarly,

493 the vector control strain MGH21 $\Delta$ cas(pVector) was generated through co-transforming two

494 empty vectors, pSmart LC KN and pBAD33Gm, into MGH21 $\Delta$ cas strain. When mutation

495 frequencies of $\mathrm{MGH} 21 \Delta \operatorname{cas}(\mathrm{pCas})$ and $\mathrm{MGH} 21 \Delta$ cas(pVector) with ertapenem were measured in

496 MHB medium supplemented with $1 \%$ arabinose (to induce the expression of casABECD),

497 kanamycin $(25 \mu \mathrm{g} / \mathrm{ml})$ and gentamicin $(10 \mu \mathrm{g} / \mathrm{ml})$.

\section{Plasmids extraction and sequencing from UCl38}

499 Plasmids from UCI38 were extracted using QIAfilter Plasmid Midi Kit (Qiagen, Cat.\# 12243).

500 Extracted plasmids were then transformed into other clinical isolates and MGH21 $\Delta$ cas through

501 electroporation. Transformants were selected on LB agar plates supplemented with cefotaxime

502 at the concentration of $10 \mu \mathrm{g} / \mathrm{ml}$. The extracted plasmid DNA was sequenced, assembled and

503 annotated as described before ${ }^{8}$.

504 Analysis of 267 K. pneumoniae genomes

505 We used a total of 267 K. pneumoniae assemblies generated at the Broad for this analysis,

506 including 80 ST258 strains. K. pneumoniae isolates were sequenced, assembled, and

507 annotated as described before ${ }^{8}$. To improve resistance gene predictions, the original gene calls 
508 from each assembly were searched against the following databases using BLAST ${ }^{53}:$ i)

509 Resfinder ${ }^{54}$ (downloaded January 23, 2018); ii) the National Database of Antibiotic Resistant

510 Organisms (https://www.ncbi.nlm.nih.gov/pathogens/antimicrobial-resistance/; downloaded

511 January 22, 2018); and iii) an in-house database of carbapenemases and ESBLs ${ }^{8}$. For each

512 gene, the database hit with the highest bit score having an e-value $<10^{-10}$ and gene length

513 coverage $>=80 \%$ was retained. The numbers of annotated carbapenemases and ß-lactamases,

514 including extended-spectrum and broad-spectrum B-lactamases, were quantified and tabulated

515 for each strain.

\section{Annotation of Restriction Modification Systems}

517 We downloaded a total of seven reference gene sets for type I $(n=3)$, type II $(n=2)$, and type III

$518(\mathrm{n}=2)$ restriction-modification systems from REBASE

519 (http://rebase.neb.com/rebase/rebase.seqs.html) on May 22, 2019. We used blastn to search

520 for these reference genes in all 267 K. pneumoniae assemblies, using an e-value cutoff of $10^{-10}$

521 and requiring $80 \%$ coverage of the reference gene. We retained the top blast hit for each

522 reference gene set and strain. We considered a restriction modification system of a certain type

523 to be present in a given strain if at least one gene from each of the two (for types II and III) or

524 three (for type I) reference sets were present in the strain.

\section{Annotation of CRISPR Arrays and cas genes}

526 CRISPR Detect ${ }^{55}$ version 2.2 was used to detect CRISPR arrays in the 267 K. pneumoniae

527 assemblies and 2453 K. pneumoniae strains available in the NCBI database using default

528 parameters. Cas genes were identified using the Broad Institute's microbial annotation

529 pipeline. For the CRISPR arrays identified in MGH21, spacer sequences were aligned to a

530 curated database of plasmid sequences ${ }^{41}$ containing sequences of 6642 plasmids, using blastn

531 and requiring with $>80 \%$ identity and coverage. Then the sequences of plasmids containing the 
532 spacer-hit genes were extracted. ResFinder ${ }^{54}$ was used to identify antibiotic resistance genes in

533 these plasmids, if any, requiring $>95 \%$ identity and $80 \%$ coverage.

\section{Determination of MICs}

535 MICs were determined by the broth microdilution method as described ${ }^{56}$. The MICs were

536 measured in duplicates in MHB medium, with a final inoculum size of $5 \times 10^{5} \mathrm{cell} / \mathrm{s} / \mathrm{ml}$

\section{Quantification of transposon insertions and SNPs in ompK36}

538 Following the robotic, modified Luria-Delbrück experiment with ertapenem treatment, 50-100

539 resistant mutants from each strain were isolated and streaked on LB agar plates supplemented

540 with ertapenem at the concentration of $1.1 \times$ MIC against the ancestor strain. Colony PCR was

541 performed using primers listed in Supplementary Table 13 to amplify ompK36 locus including

542 upstream 500 bp region of each mutant. The PCR products were then purified and Sanger

543 sequenced. Sequences were aligned to the genomic sequences of the ancestor strains and

544 single-nucleotide variants (SNVs) and transposon insertions could thus be quantified.

\section{WGS and variant calling}

546 Genomic DNA was isolated using DNeasy Blood \& Tissue Kits (Qiagen, cat.\# 69504) and

547 quantified using Qubit dsDNA HS Assay Kit (Invitrogen, cat. \# Q32851). WGS libraries were

548 made using Nextera XT DNA library preparation kit (Illumina, cat.\# FC-131-1096). Then the

549 samples were sequenced using the MiSeq or NextSeq system with 300 cycles, pair-ended. For

550 each strain sequencing depth was set at approximately $100 x$ coverage. BWA mem version

$551 \quad 0.7 .12^{57}$, and Pilon v1.23, using default settings ${ }^{58}$, were used to align reads against a reference

552 genome assembly and to identify variants, respectively. SNP positions having mapping quality

553 less than $10(\mathrm{MQ}<10)$ were not considered. The Klebsiella pneumoniae MGH21, and UCI38 
554 genome assemblies were used as references for variant identification for mutants derived from

555 each respective strain.

RNA extraction and RT-qPCR

557 Cells were cultivated in MHB or LB medium at $37{ }^{\circ} \mathrm{C}$ until early-exponential growth phase. RNA

558 was purified using Direct-zol RNA Kits (Zymo research, cat.\# R2070) and quantified with

559 Nanodrop spectrophotometer (ThermoFisher). RT-qPCR was performed using iTaq Universal

560 One-Step RT-qPCR Kits (Bio-Rad, cat.\# 1725150). RT-qPCR primers were designed using

561 Primer3 ${ }^{59}$ and are listed in Supplementary Table 13. The results were normalized as the

562 percentages of 16 rRNA.

\section{Reversion of transposon-insertion mutants and growth curves}

564 To check the reverting events of transposon insertion mutants, Mut41, Mut_UCI22, Mut_UCI43,

565 and Mut_UCl44 were cultured in replicates in LB medium with or without ertapenem $(1 \mu \mathrm{g} / \mathrm{ml})$

566 were set up and diluted every day. Each day, an aliquot of culture $(10 \mu \mathrm{L})$ from each

567 strain/replicate were diluted and plated on LB agar plates to quantify cell numbers. Colony PCR

568 was performed in 24 randomly selected colonies for PCR amplification of the ompK36 locus,

569 including 500 bp upstream and 100 bp downstream regions. The PCR product was run in

570 agarose gels to assess the size and subsequently Sanger sequenced. One revertant from

571 Mut41 was used for subsequent growth experiment and RT-qPCR to measure the expression of

572 ompK36. Growth of UCI38, Mut41 and Mut41_revertant were monitored in a Tecan plate reader

573 in LB medium at $37^{\circ} \mathrm{C}$ for 8 hours. All experiments were repeated three times.

\section{Conjugation}

575 Rifampin mutants of UCI38, Mut34, Mut101, Mut186 and Mut195 were raised by plating the

576 exponential-growth phase cells on LB agar plates containing $50 \mu \mathrm{g} / \mathrm{mL}$ rifampin. After overnight 
577 incubation, rifampin mutants from each strain were selected and subjected to WGS. Mutants

578 that only have mutations in $r p o B$ were selected for conjugation. Exponential growth phase cells

579 of rifampin mutants from these five strains were mixed with BIDMC45 cells at $1: 1$ ratio, then the

580 mixture was spotted on LB agar plates without antibiotics or containing meropenem $(0.003$

$581 \mu \mathrm{g} / \mathrm{ml}$ ) and grown overnight. The second day morning, cells were transferred to LB liquid

582 medium, serial diluted, and plated on LB agar plates containing meropenem $(2 \mu \mathrm{g} / \mathrm{ml})$ and

583 rifampin $(50 \mu \mathrm{g} / \mathrm{ml})$ for the selection of conjugants. Meanwhile, diluted cells were plated on

584 rifampin $(50 \mu \mathrm{g} / \mathrm{ml})$ plates to quantify cell concentrations. All experiments were repeated three

585 time.

Data Availability

587 All data generated or analyzed during this study are included in this article and in the 588 supplementary tables. Sequencing data is deposited to NCBI under the accession number 589 PRJNA670748.

\section{Acknowledgments}

591 We thank James Gomez for instructions and suggestions of setting up the modified Luria-

592 Delbrück experiment and his further input and comments on this project. We thank Noam

593 Shoresh for the valuable discussions on the data analysis of the modified Luria-Delbrück. We

594 thank Sharon Wong, Anne Clatworthy and Thulasi Warrier for their comments on the

595 manuscript. This publication was supported in part by the National Institute of Allergy and

596 Infectious Diseases of the National Institutes of Health under award 5R01 Al117043-05 to DTH

597 and U19Al110818 to the Broad Institute, and by a generous gift from Anita and Josh

598 Bekenstein. 
601 P.M. and D.T.H. designed the study and wrote the manuscript. P.M., L.L.H., and H.H.L.

602 performed the experiments. C.M.E. provided suggestions on the initial isolate selection for these

603 experiments and provided critical input on the manuscript preparation. R.P.B. provided

604 extensive input and suggestions on the design of this study and manuscript preparation. A.P.

605 did genomic analysis in the assemblies of 267 K. pneumoniae isolates and 2453 K. pneumoniae

606 genomes available in the NCBI database. A.L.M. analyzed the WGS data of mutants and

607 identified mutations in each mutant. A.M.E. supervised the genomic data analysis and SNP

608 identification, and provided extensive input on the design of the genomic analysis. J.L. analyzed

609 RNA-seq data. All authors have read and approved the manuscript.

610

611 Competing Interests statement

612 The authors declare no competing interests.

\section{References}

1. MacLean, R.C. \& San Millan, A. The evolution of antibiotic resistance. Science 365, 1082-1083 (2019).

2. Marraffini, L.A. \& Sontheimer, E.J. CRISPR interference limits horizontal gene transfer in staphylococci by targeting DNA. Science 322, 1843-5 (2008).

3. Rosenberg, S.M. Evolving responsively: adaptive mutation. Nat Rev Genet 2, 504-15 (2001).

4. Cirz, R.T. et al. Inhibition of mutation and combating the evolution of antibiotic resistance. PLoS Biol 3, e176 (2005).

5. Blazquez, J., Rodriguez-Beltran, J. \& Matic, I. Antibiotic-Induced Genetic Variation: How It Arises and How It Can Be Prevented. Annu Rev Microbiol 72, 209-230 (2018).

6. Papp-Wallace, K.M., Endimiani, A., Taracila, M.A. \& Bonomo, R.A. Carbapenems: past, present, and future. Antimicrob Agents Chemother 55, 4943-60 (2011).

7. Zhanel, G.G. et al. Comparative review of the carbapenems. Drugs 67, 1027-52 (2007).

8. Cerqueira, G.C. et al. Multi-institute analysis of carbapenem resistance reveals remarkable diversity, unexplained mechanisms, and limited clonal outbreaks. Proc Natl Acad Sci U S A 114, 1135-1140 (2017).

9. Ma, P., Laibinis, H.H., Ernst, C.M. \& Hung, D.T. Carbapenem Resistance Caused by High-Level Expression of OXA-663 beta-Lactamase in an OmpK36-Deficient Klebsiella pneumoniae Clinical Isolate. Antimicrob Agents Chemother 62(2018).

10. Poirel, L., Pitout, J.D. \& Nordmann, P. Carbapenemases: molecular diversity and clinical consequences. Future Microbiol 2, 501-12 (2007). 
636

637

638

639

640

641

642

643

644

645

646

647

648

649

650

651

652

653

654

655

656

657

658

659

660

661

662

663

664

665

666

667

668

669

670

671

672

673

674

675

676

677

678

679

680

681

682

683

684

11. Martinez-Martinez, L. et al. Roles of beta-lactamases and porins in activities of carbapenems and cephalosporins against Klebsiella pneumoniae. Antimicrob Agents Chemother 43, 1669-73 (1999).

12. Navon-Venezia, S., Kondratyeva, K. \& Carattoli, A. Klebsiella pneumoniae: a major worldwide source and shuttle for antibiotic resistance. FEMS Microbiol Rev 41, 252-275 (2017).

13. Wyres, K.L., Lam, M.M.C. \& Holt, K.E. Population genomics of Klebsiella pneumoniae. Nat Rev Microbiol 18, 344-359 (2020).

14. Deleo, F.R. et al. Molecular dissection of the evolution of carbapenem-resistant multilocus sequence type 258 Klebsiella pneumoniae. Proc Natl Acad Sci U S A 111, 4988-93 (2014).

15. Bowers, J.R. et al. Genomic Analysis of the Emergence and Rapid Global Dissemination of the Clonal Group 258 Klebsiella pneumoniae Pandemic. PLoS One 10, e0133727 (2015).

16. Pitout, J.D., Nordmann, P. \& Poirel, L. Carbapenemase-Producing Klebsiella pneumoniae, a Key Pathogen Set for Global Nosocomial Dominance. Antimicrob Agents Chemother 59, 5873-84 (2015).

17. Munoz-Price, L.S. et al. Clinical epidemiology of the global expansion of Klebsiella pneumoniae carbapenemases. Lancet Infect Dis 13, 785-96 (2013).

18. Wyres, K.L. et al. Genomic surveillance for hypervirulence and multi-drug resistance in invasive Klebsiella pneumoniae from South and Southeast Asia. Genome Med 12, 11 (2020).

19. Rada, A.M. et al. Dynamics of bla KPC-2 dissemination from non-CG258 Klebsiella pneumoniae to other Enterobacterales via IncN plasmids in an area of high endemicity. Antimicrob Agents Chemother (2020).

20. Marsh, J.W. et al. Evolution of Outbreak-Causing Carbapenem-Resistant Klebsiella pneumoniae ST258 at a Tertiary Care Hospital over 8 Years. mBio 10(2019).

21. Bonnin, R.A. et al. Emergence of New Non-Clonal Group 258 High-Risk Clones among Klebsiella pneumoniae Carbapenemase-Producing K. pneumoniae Isolates, France. Emerg Infect Dis 26, 1212-1220 (2020).

22. Mathers, A.J. et al. Molecular dissection of an outbreak of carbapenem-resistant enterobacteriaceae reveals Intergenus KPC carbapenemase transmission through a promiscuous plasmid. mBio 2, e00204-11 (2011).

23. Strydom, K.A. et al. Klebsiella pneumoniae ST307 with OXA-181: threat of a high-risk clone and promiscuous plasmid in a resource-constrained healthcare setting. $J$ Antimicrob Chemother 75, 896-902 (2020).

24. Peirano, G., Chen, L., Kreiswirth, B.N. \& Pitout, J.D.D. Emerging Antimicrobial-Resistant High-Risk Klebsiella pneumoniae Clones ST307 and ST147. Antimicrob Agents Chemother 64(2020).

25. Chen, L. et al. Carbapenemase-producing Klebsiella pneumoniae: molecular and genetic decoding. Trends Microbiol 22, 686-96 (2014).

26. Eilertson, B. et al. CG258 Klebsiella pneumoniae isolates without beta-lactam resistance at the onset of the carbapenem-resistant Enterobacteriaceae epidemic in New York City. $J$ Antimicrob Chemother 74, 17-21 (2019).

27. Li, H.Y. et al. Characterization of CRISPR-Cas Systems in Clinical Klebsiella pneumoniae Isolates Uncovers Its Potential Association With Antibiotic Susceptibility. Front Microbiol 9, 1595 (2018).

28. Mackow, N.A. et al. CRISPR-Cas influences the acquisition of antibiotic resistance in Klebsiella pneumoniae. PLoS One 14, e0225131 (2019). 
685

686

687

688

689

690

691

692

693

694

695

696

697

698

699

700

701

702

703

704

705

706

707

708

709

710

711

712

713

714

715

716

717

718

719

720

721

722

723

724

725

726

727

728

729

730

731

732

733

734

29. Tang, Y. et al. Absence of the type I-E CRISPR-Cas system in Klebsiella pneumoniae clonal complex 258 is associated with dissemination of IncF epidemic resistance plasmids in this clonal complex. J Antimicrob Chemother 75, 890-895 (2020).

30. Gandra, S., Klein, E.Y., Pant, S., Malhotra-Kumar, S. \& Laxminarayan, R. Faropenem Consumption is Increasing in India. Clin Infect Dis 62, 1050-2 (2016).

31. Queenan, A.M., Shang, W., Flamm, R. \& Bush, K. Hydrolysis and inhibition profiles of beta-lactamases from molecular classes $A$ to $D$ with doripenem, imipenem, and meropenem. Antimicrob Agents Chemother 54, 565-9 (2010).

32. Kohler, J. et al. In vitro activities of the potent, broad-spectrum carbapenem MK-0826 (L$749,345)$ against broad-spectrum beta-lactamase-and extended-spectrum betalactamase-producing Klebsiella pneumoniae and Escherichia coli clinical isolates. Antimicrob Agents Chemother 43, 1170-6 (1999).

33. Sutaria, D.S. et al. First Penicillin-Binding Protein Occupancy Patterns of beta-Lactams and beta-Lactamase Inhibitors in Klebsiella pneumoniae. Antimicrob Agents Chemother 62(2018).

34. Tsai, Y.K. et al. Effects of different resistance mechanisms on susceptibility to different classes of antibiotics in Klebsiella pneumoniae strains: a strategic system for the screening and activity testing of new antibiotics. J Antimicrob Chemother 72, 3302-3316 (2017).

35. Jones, R.N., Sader, H.S. \& Fritsche, T.R. Comparative activity of doripenem and three other carbapenems tested against Gram-negative bacilli with various beta-lactamase resistance mechanisms. Diagn Microbiol Infect Dis 52, 71-4 (2005).

36. Martinez, J.L. \& Baquero, F. Mutation frequencies and antibiotic resistance. Antimicrob Agents Chemother 44, 1771-7 (2000).

37. Gomez, J.E. et al. Ribosomal mutations promote the evolution of antibiotic resistance in a multidrug environment. Elife 6(2017).

38. Goldstein, B.P. Resistance to rifampicin: a review. J Antibiot (Tokyo) 67, 625-30 (2014).

39. Pope, C.F., O'Sullivan, D.M., McHugh, T.D. \& Gillespie, S.H. A practical guide to measuring mutation rates in antibiotic resistance. Antimicrob Agents Chemother 52, 1209-14 (2008).

40. Bujnicki, J.M. \& Radlinska, M. Molecular evolution of DNA-(cytosine-N4) methyltransferases: evidence for their polyphyletic origin. Nucleic Acids Res 27, 4501-9 (1999).

41. Brooks, L., Kaze, M. \& Sistrom, M. A Curated, Comprehensive Database of Plasmid Sequences. Microbiol Resour Announc 8(2019).

42. Nicolas-Chanoine, M.H., Mayer, N., Guyot, K., Dumont, E. \& Pages, J.M. Interplay Between Membrane Permeability and Enzymatic Barrier Leads to Antibiotic-Dependent Resistance in Klebsiella Pneumoniae. Front Microbio/ 9, 1422 (2018).

43. Mahillon, J. \& Chandler, M. Insertion sequences. Microbiol Mol Biol Rev 62, 725-74 (1998).

44. Knopp, M. \& Andersson, D.I. Amelioration of the Fitness Costs of Antibiotic Resistance Due To Reduced Outer Membrane Permeability by Upregulation of Alternative Porins. Mol Biol Evol 32, 3252-63 (2015).

45. Phan, K. \& Ferenci, T. The fitness costs and trade-off shapes associated with the exclusion of nine antibiotics by OmpF porin channels. ISME J11, 1472-1482 (2017).

46. Miller, C. et al. SOS response induction by beta-lactams and bacterial defense against antibiotic lethality. Science 305, 1629-31 (2004).

47. Nicoloff, H., Hjort, K., Levin, B.R. \& Andersson, D.I. The high prevalence of antibiotic heteroresistance in pathogenic bacteria is mainly caused by gene amplification. Nat Microbiol 4, 504-514 (2019). 
48. Andersson, D.I., Nicoloff, H. \& Hjort, K. Mechanisms and clinical relevance of bacterial heteroresistance. Nat Rev Microbiol 17, 479-496 (2019).

49. Rodriguez-Bano, J., Gutierrez-Gutierrez, B., Machuca, I. \& Pascual, A. Treatment of Infections Caused by Extended-Spectrum-Beta-Lactamase-, AmpC-, and Carbapenemase-Producing Enterobacteriaceae. Clin Microbiol Rev 31(2018).

50. Zheng, Y., Liu, X.P. \& Liu, J.H. [Highly efficient transformation with plasmid DNA in Klebsiella pneumoniae]. Wei Sheng Wu Xue Bao 47, 721-4 (2007).

51. Link, A.J., Phillips, D. \& Church, G.M. Methods for generating precise deletions and insertions in the genome of wild-type Escherichia coli: application to open reading frame characterization. J Bacteriol 179, 6228-37 (1997).

52. Guzman, L.M., Belin, D., Carson, M.J. \& Beckwith, J. Tight regulation, modulation, and high-level expression by vectors containing the arabinose PBAD promoter. J Bacteriol 177, 4121-30 (1995).

53. Altschul, S.F., Gish, W., Miller, W., Myers, E.W. \& Lipman, D.J. Basic local alignment search tool. J Mol Biol 215, 403-10 (1990).

54. Zankari, E. et al. Identification of acquired antimicrobial resistance genes. J Antimicrob Chemother 67, 2640-4 (2012).

55. Biswas, A., Staals, R.H., Morales, S.E., Fineran, P.C. \& Brown, C.M. CRISPRDetect: A flexible algorithm to define CRISPR arrays. BMC Genomics 17, 356 (2016).

56. Wiegand, I., Hilpert, K. \& Hancock, R.E. Agar and broth dilution methods to determine the minimal inhibitory concentration (MIC) of antimicrobial substances. Nat Protoc 3 , 163-75 (2008).

57. Li, H. \& Durbin, R. Fast and accurate short read alignment with Burrows-Wheeler transform. Bioinformatics 25, 1754-60 (2009).

58. Walker, B.J. et al. Pilon: an integrated tool for comprehensive microbial variant detection and genome assembly improvement. PLoS One 9, e112963 (2014).

59. Koressaar, T. \& Remm, M. Enhancements and modifications of primer design program Primer3. Bioinformatics 23, 1289-91 (2007). 
Table 1. Characterization of representative mutants resistant to both ertapenem and meropenem or to ertapenem alone.

\begin{tabular}{|c|c|c|c|c|c|}
\hline \multirow[t]{2}{*}{ Mutant ID } & \multirow[t]{2}{*}{ Ancestor } & \multirow[t]{2}{*}{$\begin{array}{l}\text { Mutations causing decreased } \\
\text { susceptibility }\end{array}$} & \multirow[t]{2}{*}{ Gene function } & \multicolumn{2}{|c|}{$\begin{array}{l}\text { Fold changes of MICs compared to } \\
\text { ancestor strains }\end{array}$} \\
\hline & & & & ETP & MEM \\
\hline \multicolumn{6}{|c|}{ Mutants resistant to ertapenem alone } \\
\hline Mut87 & MGH21 & wzc (L367_02683): c.381delA & exopolysaccharide biosynthesis & 2 & 1 \\
\hline Mut86 & MGH21 & ompA (L367_001084): p.G31D & porin & 2 & 1 \\
\hline Mut131 & MGH21 & $\begin{array}{c}\text { ompK36 (L367_002817), } \\
\text { c.332_333insTACGACCGTAGT }\end{array}$ & porin & 256 & 1 \\
\hline Mut101 & UCI38 & rseA (P841_001338):: IS1 & $\begin{array}{l}\text { anti-sigma } E \text { factor, involved in } \\
\text { maintaining cell envelop integrity }\end{array}$ & 8 & 1 \\
\hline Mut34 & UCI38 & blasHv-12 (P841_005417) dup[2] & ESBL & 8 & 1 \\
\hline \multicolumn{6}{|c|}{ Mutants resistant to ertapenem and meropenem } \\
\hline Mut21 & MGH21 & bamD (L367_003146): c.-4C>T & $\begin{array}{l}\text { outer membrane protein } \\
\text { assembly factor }\end{array}$ & 8 & 2 \\
\hline Mut136 & MGH21 & omp36K (L367_002817): p.Q311STP & porin & 64 & 8 \\
\hline Mut41 & UCI38 & ompK36 (P841_001022):: IS1 & porin & 64 & 8 \\
\hline Mut107 & UCI38 & ompK36 (P841_001022):: IS5 & porin & 64 & 16 \\
\hline Mut45 & UCI38 & ompK36 (P841_001022):: IS91 & porin & 64 & 16 \\
\hline
\end{tabular}



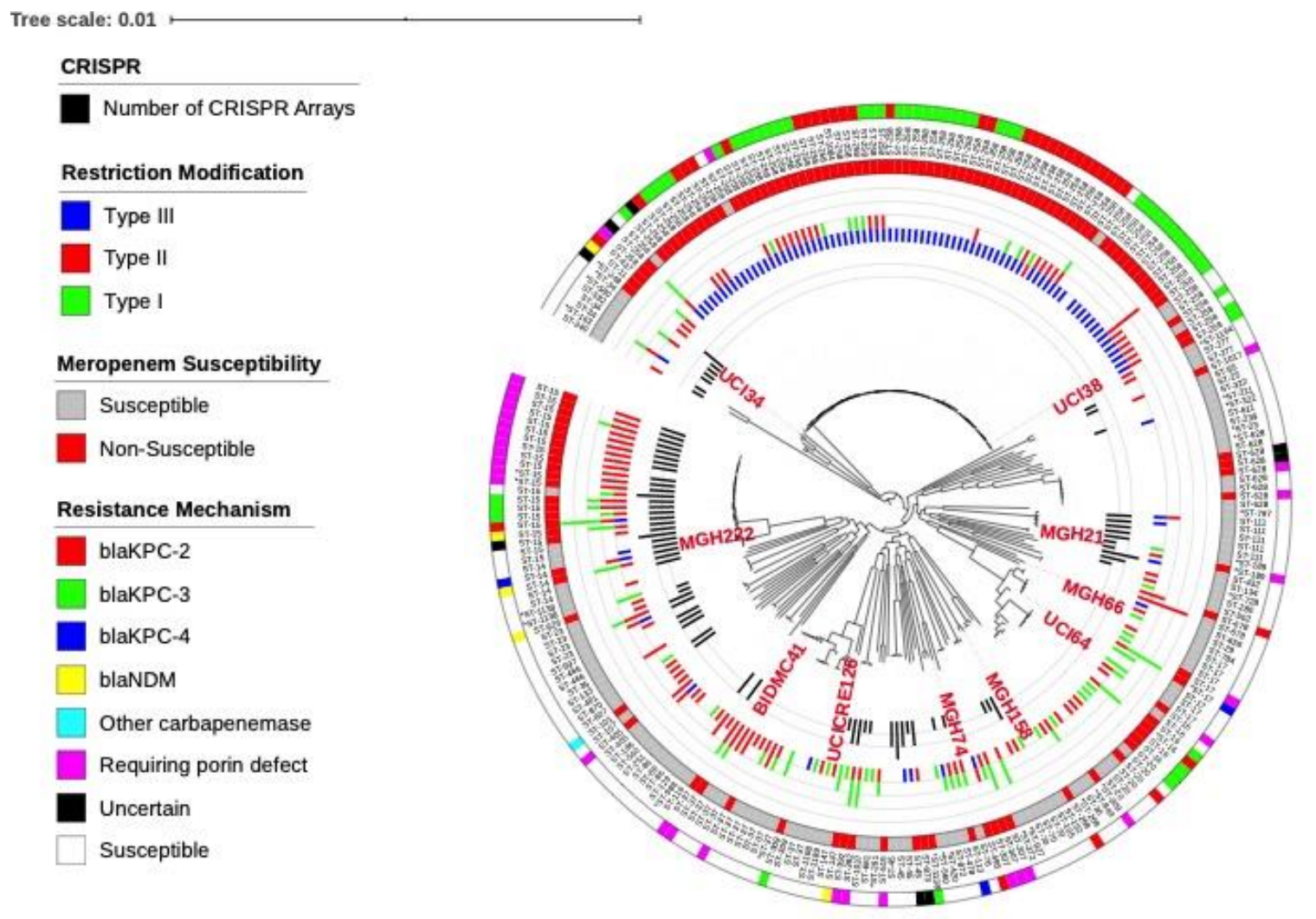

Fig. 1 Ten phylogenetically diverse carbapenem-susceptible $K$. pneumoniae isolates were selected from a collection of 267 K. pneumoniae clinical isolates. The selected isolates are highlighted in red. In this phylogenetic tree, from inner to outer circles, the content of the CRISPR-Cas systems, Restriction-Modification systems, susceptibility to carbapenems, and sequence types are indicated. For carbapenem-resistant isolates, the resistance mechanism is also indicated. 
a

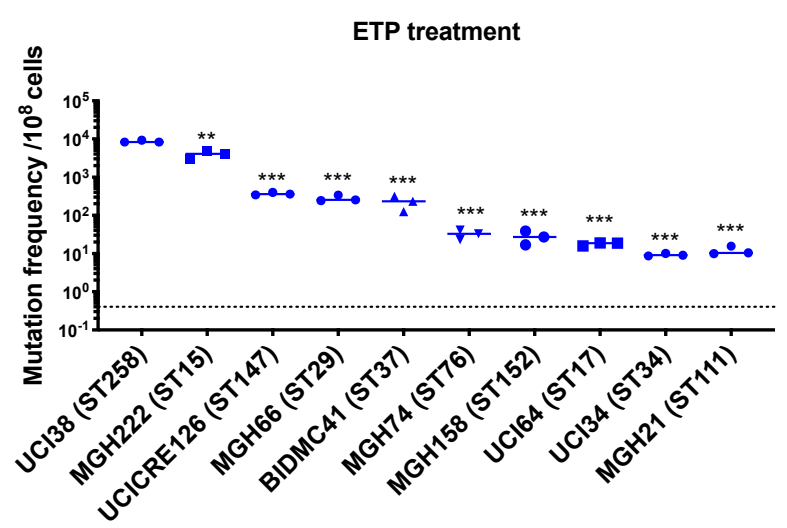

C

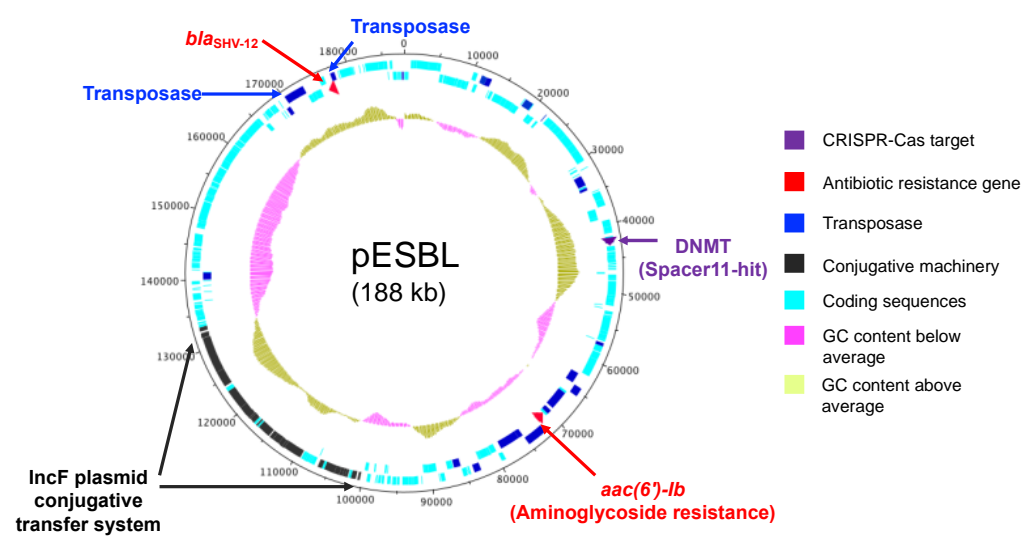

b

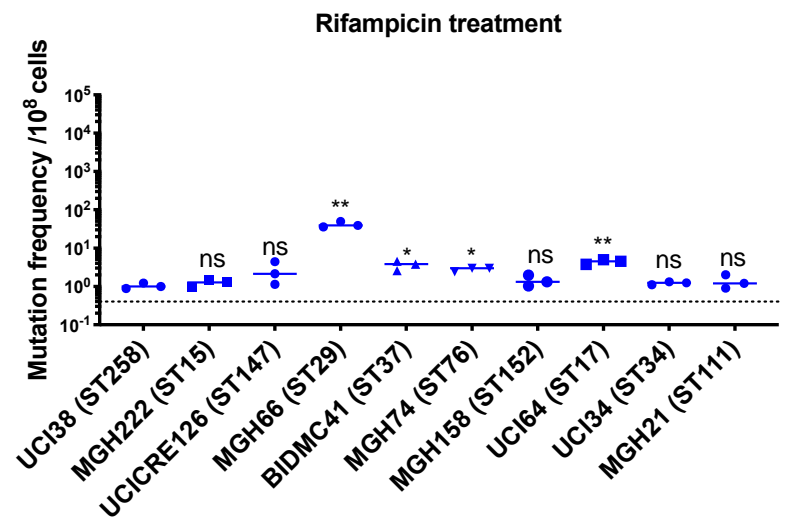

d

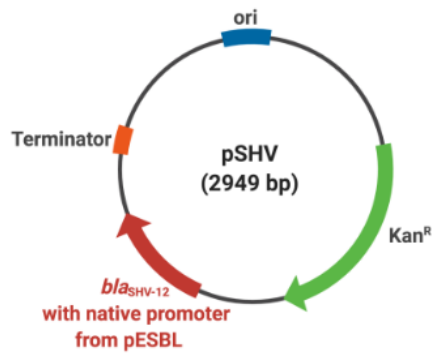

e

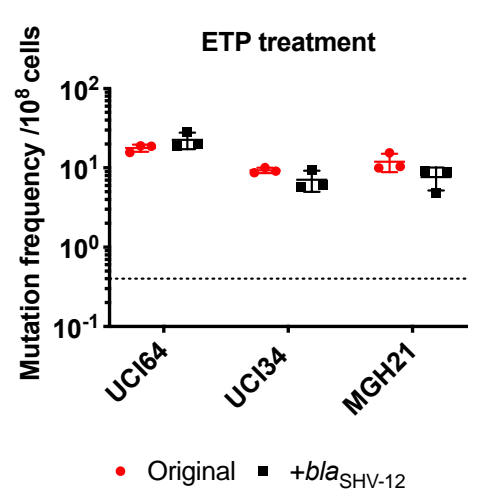

f

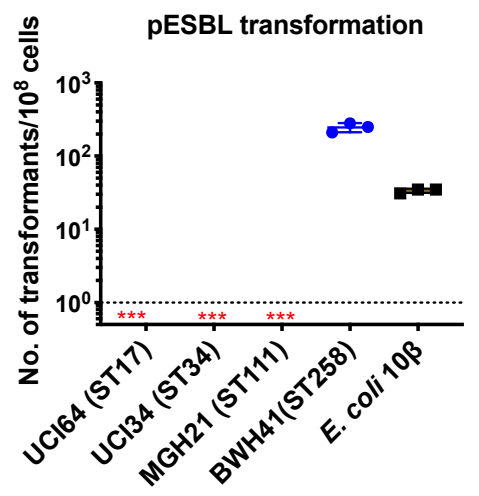

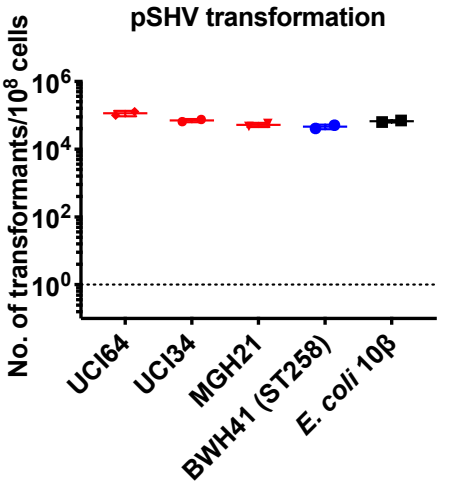

Fig. 2 The evolution of carbapenem resistance is affected by genetic background of the

isolates. a, Mutation frequencies of ten clinical isolates under treatment with ertapenem. Five isolates, UCI38 (ST258), MGH222 (ST15), UCICRE126 (ST147), MGH66 (ST29), and 
BIDMC41 (ST37) have relatively greater mutation frequencies to ertapenem (>100 mutants per $10^{8}$ cells) than the other five isolates. Comparing to UCI38 (ST258) which has the highest mutation frequencies to ertapenem, all isolates have significantly different mutation frequencies. Two-tailed Student's t-test was used for statistical analysis between UCI38 (ST258) and other isolates. $\mathbf{b}$, Mutation frequencies of ten clinical isolates under treatment with rifampicin. Isolates with relatively high-level mutation frequencies to ertapenem do not necessarily have high-level mutation frequencies to rifampicin. Two-tailed Student's t-test was used for statistical analysis between UCI38 (ST258) and other isolates. c, Diagram of pESBL, an ESBL-encoding plasmid isolated from UCI38 (ST258). d, Diagram of pSHV, a multi-copy laboratory plasmid containing the native promoter and coding region of the ESBL gene blasHV-12 amplified from pESBL. e, The ESBL gene, blasHv-12, was amplified from pESBL and expressed in three isolates lacking an ESBL gene and with relatively low-level mutation frequencies to ertapenem. However, mutation frequencies to ertapenem were not changed compared to the original strains lacking an ESBL gene (red). Two-tailed Student's t-test was used for statistical analysis to compare the original strain with the corresponding strain overexpressing blasHV-12, with $p>0.05$ for all three pairs. $\mathbf{f}$, Transformation efficiencies of pESBL(left) or pSHV (right) in three isolates lacking ESBL genes (red) and with relatively low-level mutation frequencies to ertapenem. As controls, these two plasmids were also transformed into another ST258 strain BWH41 (blue) which does not carry ESBL genes, and a strain of E. coli $10 \beta$ (black). pESBL could not be transformed into these three isolates but it could be transformed into BWH41 (ST258) and E. coli. In contrast, the laboratory construct pSHV was successfully transformed into all strains tested. For all experiments in $\mathbf{a}, \mathbf{b}, \mathbf{e}, \mathbf{f}$, two to three independent biological replicates were performed. Data from independent experiments were plotted individually with error bars plotted as the standard deviation. The limit of detection is indicated with a dashed line, and the asterisk $\left({ }^{*}\right)$ under the dashed line indicates frequencies under the limit of detection. ${ }^{*} p<0.05 ;{ }^{* *} p<0.005 ;{ }^{* * *} p<$ 0.0005; ns, not significant. 


\section{a}

Type I-E CRISPR-Cas system in MGH21

CRISPR array II

CRISPR array I

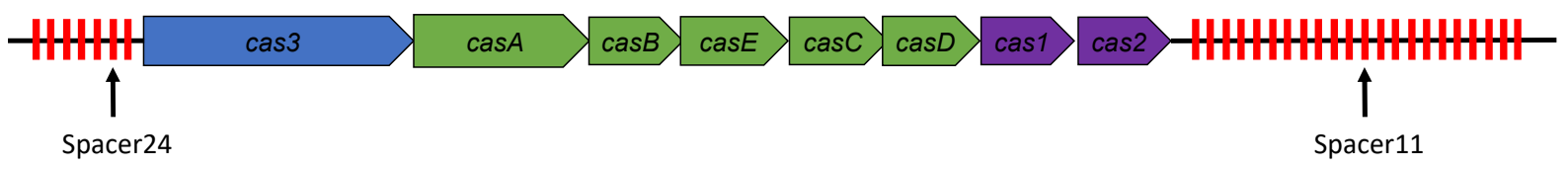

b

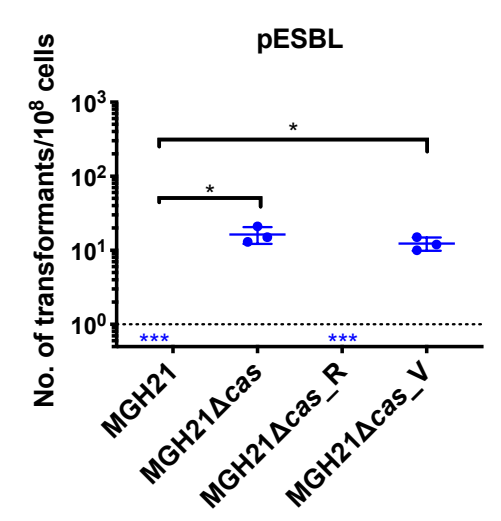

C

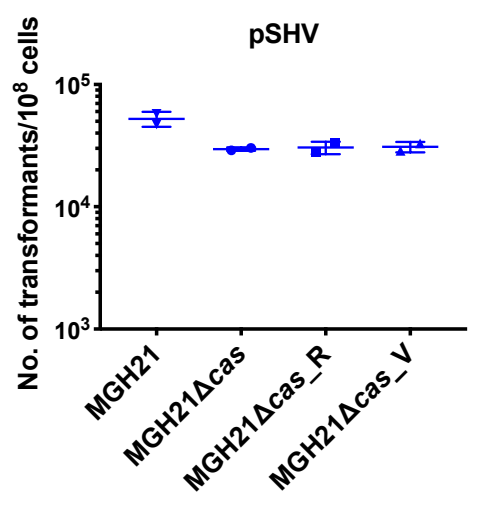

d

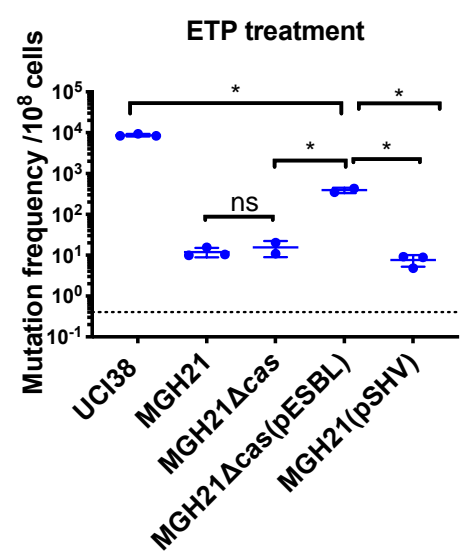

Fig. 3 Type I-E CRISPR-Cas system in MGH21 (ST111) prevents the acquisition of pESBL but the presence of pESBL alone does not account for high mutation frequencies. a, Type I-E CRISPR-Cas system in MGH21 (ST111). The two CRISPR arrays and the position of two spacers (Spacer11 and Spacer24) that align to plasmids encoding resistance genes are indicated. Spacer11 aligns to the DNMT gene located on pESBL (Fig. 2C). b-c, Transformation efficiencies of pESBL (b) or the lab construct pSHV (c) in MGH21, MGH21 1 cas, MGH21 1 cas(pCas) with CRISPR-Cas complementation, and MGH21 $\Delta$ cas(pVector) with control vector complementation. pESBL could only be transformed into MGH21 strains in which the CRISPR-Cas system was deleted (MGH21 $\Delta$ cas and MGH21 $\Delta$ cas (pVector)), whereas pSHV could be transformed into all strains at similar efficiencies. d, Mutation frequencies of UCI38 (ST258), MGH21, MGH21 $\Delta$ cas, MGH21 $\operatorname{cas}(\mathrm{pESBL})$, and MGH21(pSHV) with ertapenem treatment. The deletion of the CRISPR-Cas system (MGH21 $\Delta$ cas) and the introduction of pSHV $(\mathrm{MGH} 21(\mathrm{pSHV}))$ did not affect the mutation frequencies. In contrast, the introduction of $\mathrm{pESBL}$ 
$(\mathrm{MGH} 21 \Delta \operatorname{cas}(\mathrm{pESBL}))$ increased mutation frequencies, indicating that some factors on $\mathrm{pESBL}$ other than the ESBL gene affect the mutation frequencies. However, mutations frequencies of $\mathrm{MGH} 21 \Delta \operatorname{cas}(\mathrm{pESBL})$ were still significantly lower than these of UCl38, indicating that more factors in the genetic background of UCI38 contribute to the high-level mutation frequencies. All experiments were performed in triplicate and data were plotted individually. Error bars were plotted as standard deviation. The limit of detection of each assay is indicated with a dashed line, and the asterisk $\left({ }^{*}\right)$ under the dashed line indicates that the transformation efficiencies are below the limit of detection. Two-tailed Student's t-test was used for all statistical analysis; an asterisk marking a pair-wise comparison denotes a $p<0.05$. 
a

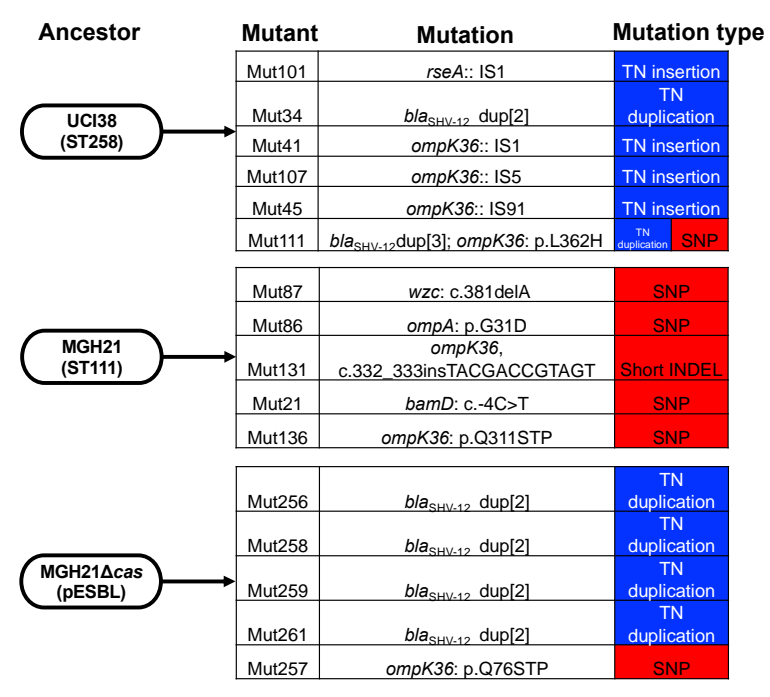

b

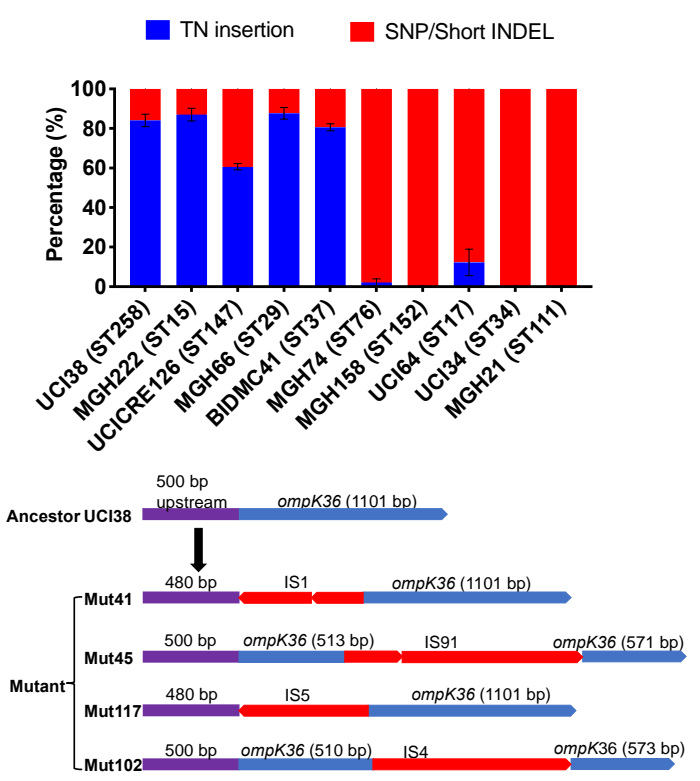

d

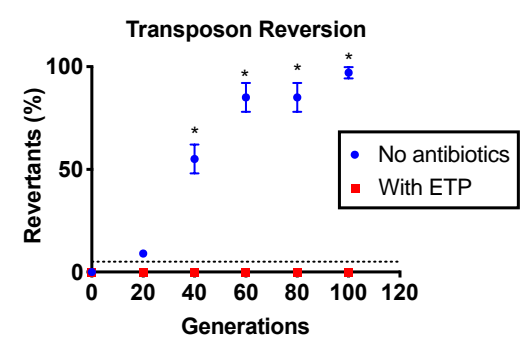

e

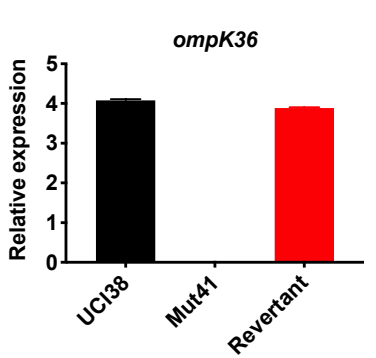

f

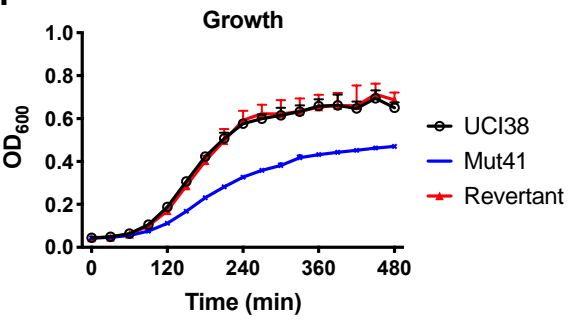

Fig. 4 Transposon insertional mutagenesis causes frequent and reversible inactivation of porin genes in isolates with high-level mutation frequencies to ertapenem. a, Mutation types (transposon insertion/duplication (blue) vs. SNP (red)) identified via WGS in ertapenem resistant mutants of UCl38 (ST258), MGH21 (ST111), and MGH21 $\Delta$ cas(pESBL). The majority of mutants that carry pESBL (UCl38 and MGH21 $\Delta \operatorname{cas}(\mathrm{pESBL}))$ have transposon-mediated mutations, while only SNPs or short insertion/deletions were observed in mutants of MGH21. b, Relative quantification of the propensity of ten selected isolates to undergo transposon insertion (blue) versus SNP acquisition (red) in ompK36 during ertapenem treatment. For each of these strains, 50-100 mutants were isolated, and the types of mutation in ompK36 locus, if any, was determined via Sanger sequencing. Transposon insertions occurred at $\sim 10$ times higher frequencies than the acquisition of SNPs or short insertion/deletion in strains with higher level of 
mutation frequencies to ertapenem. c, Illustration of ompK36 inactivation by four transposons in UCI38. Four representative mutants derived from UCI38 were selected and the insertion sites were determined by Sanger sequencing. d, Transposon disruption of ompK36 was reversible. A representative ertapenem resistant mutant with an IS1 insertion in ompK36, Mut41, was cultured in the presence or absence of ertapenem. Every 20 generations, colony PCR targeting ompK36 locus was performed on the culture to quantify the percentage of the population that had lost the transposon insertion at this locus. Two-tailed Student's t-test was used for statistical analysis at each time point to compare the cultures with and without antibiotics. e-f, The relative expression of ompK36 (e) and growth curves (f) of UCI38 (black), Mut41 (red) and one representative revertant of Mut41 (blue). All experiments were performed in triplicate. Error bars are plotted as the standard deviation. 
a
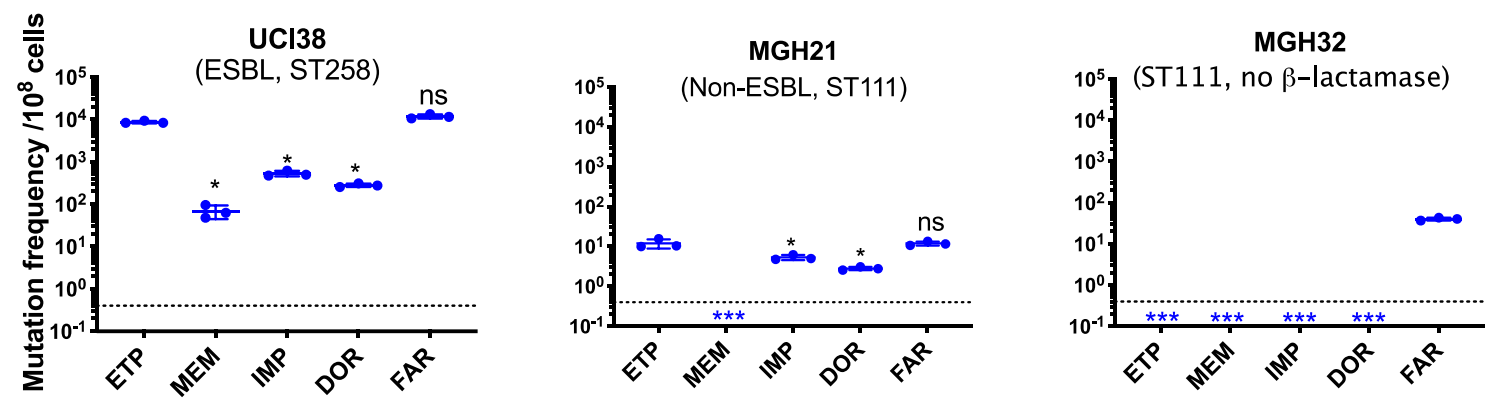

b

C

d

e
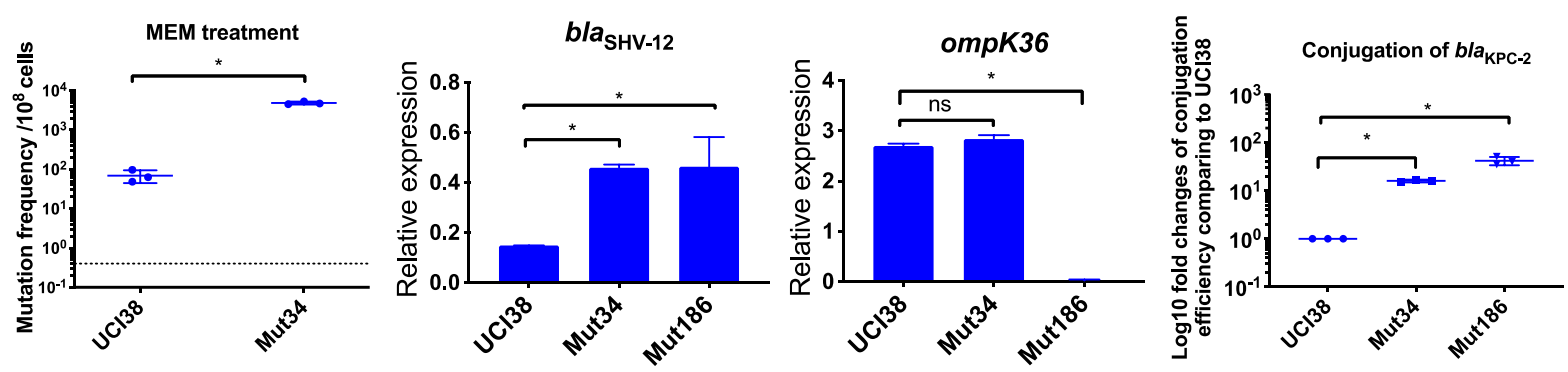

Fig. 5 Ertapenem and faropenem treatment are not only associated with higher mutation frequencies, but they also promote the evolution of meropenem resistance. a, Mutation frequencies of three representative isolates, UCI38 (ST258), MGH21 (ST111) and MGH32 (ST111, no $\beta$-lactamase), under separate treatment with ertapenem (ETP), meropenem (MEM), imipenem (IMP), doripenem (DOR), or faropenem (FAR). Higher mutation frequencies are associated with ertapenem and faropenem treatment, while lower mutation frequencies are observed with meropenem treatment. In MGH32, an isolate without $\beta$-lactamase genes, only faropenem-resistant mutants were isolated. Two-tailed student's t-test was used for statistical analysis to compare between ertapenem treatment and other carbapenems or faropenem. $\mathbf{b}$, Mutation frequencies of $\mathrm{UCI} 38$ and Mut34, an ertapenem-restricted resistant mutant derived from UCl38, under treatment with meropenem. Despite having the same MIC of meropenem as UCI38, Mut34 had higher mutation frequencies than UCI38. c-d, Relative expression levels of blasHV-12 (c) or ompK36 (d) in UCl38, Mut34, and Mut186 (an ertapenem and meropenem resistant mutant derived from Mut34) show the progressive acquisition of mutations to achieve 
meropenem resistance. Mut34 has increased blasHV-12 relative to its parent UCI38; Mut186 has disrupted ompK36, relative to its parent Mut34. e, Conjugation efficiencies of UCI38, Mut34, and Mut186 with K. pneumoniae clinical isolate BIDMC45 carrying blakPC-2. In the presence of meropenem, Mut186 had the highest conjugation efficiency with UCI38 having the lowest. All experiments were performed in triplicate. Two-tailed student's t-test was used for statistical analysis to compare UCI38 with other strains. Error bars are plotted as standard deviation. The limit of detection is indicated with a dashed line, and the asterisk $\left(^{*}\right)$ under the dashed line indicates frequencies under the limit of detection. 


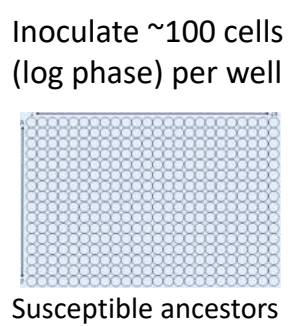

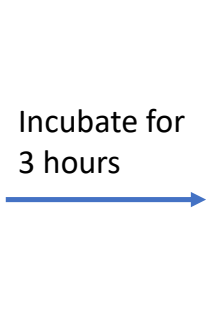

10000 cells per well, quantify by plating

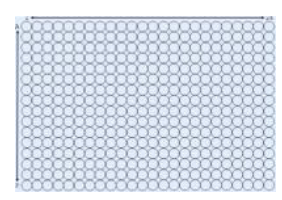

Add antibiotics
Measure OD600

Quantify positive and negative wells

Sub-culturing mutants to confirm the increase of MICS

Supplementary Fig. 1 Scheme of the modified Luria-Delbrück system. Exponential-phase growing cells are diluted to roughly 100 cells / $50 \mu$ with MHB medium and inoculated into three to six 384-well plates using Bravo automatic liquid handling platform, followed by incubation at $37^{\circ} \mathrm{C}$ for 3 hours. $10 \mu \mathrm{l}$ of culture from 3 randomly selected wells was taken to plate on LB agar plates for estimating numbers of cells in the wells. Then, antibiotics were added at the concentrations of $1.1 \times$ MICs or at specified concentrations using Bravo automatic liquid handling platform, and cultures were incubated at $37^{\circ} \mathrm{C}$ overnight. $O D_{600}$ was measured the next day and positive and negative wells were quantified. Mutants from each plate were subcultured in MHB medium supplemented with the same antibiotics at the same concentrations used for the selection, and saved in $25 \%$ glycerol stocks for future analysis. Mutants that did not grow up in the sub-culturing were excluded from the calculation of mutation frequencies. 


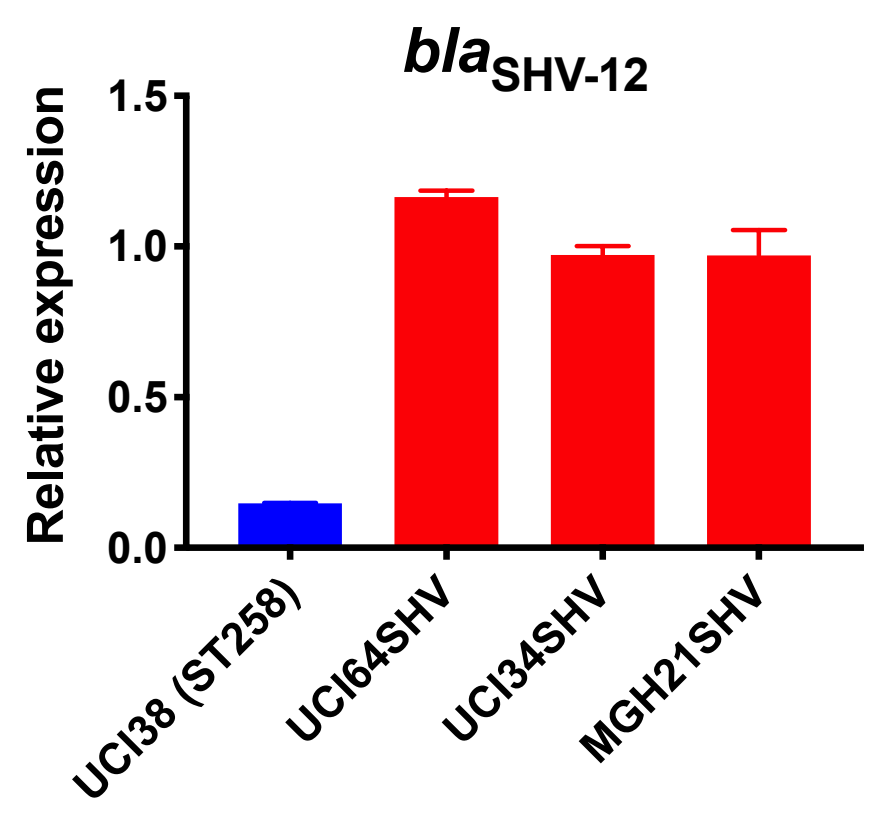

Supplementary Fig. 2 Relative expression of blasHV-12 in three strains overexpressing

blasHV-12 through pSHV. BlasHV-12, including the promoter region, was amplified from UCI38 and expressed in three strains lacking an ESBL gene. The express levels of blasHV-12 in these overexpression strains were higher than it in UCl38 because pSHV is a multi-copy plasmid. RTqPCR data was normalized to 16S rRNA. Experiments were repeated 3 times and error bars were plotted as standard deviation. 
bioRxiv preprint doi: https://doi.org/10.1101/2021.02.11.430761: this version posted Februarv 12. 2021. The copvriaht holder for this preprint (which was not certified by peer review) is the author/funder, who has granted bioRxiv a license to display the preprint in perpetuity. It is made available under aCC-BY 4.0 International license.

Type I-E CRISPR-Cas system in MGH21

CRISPR array II

CRISPR array I

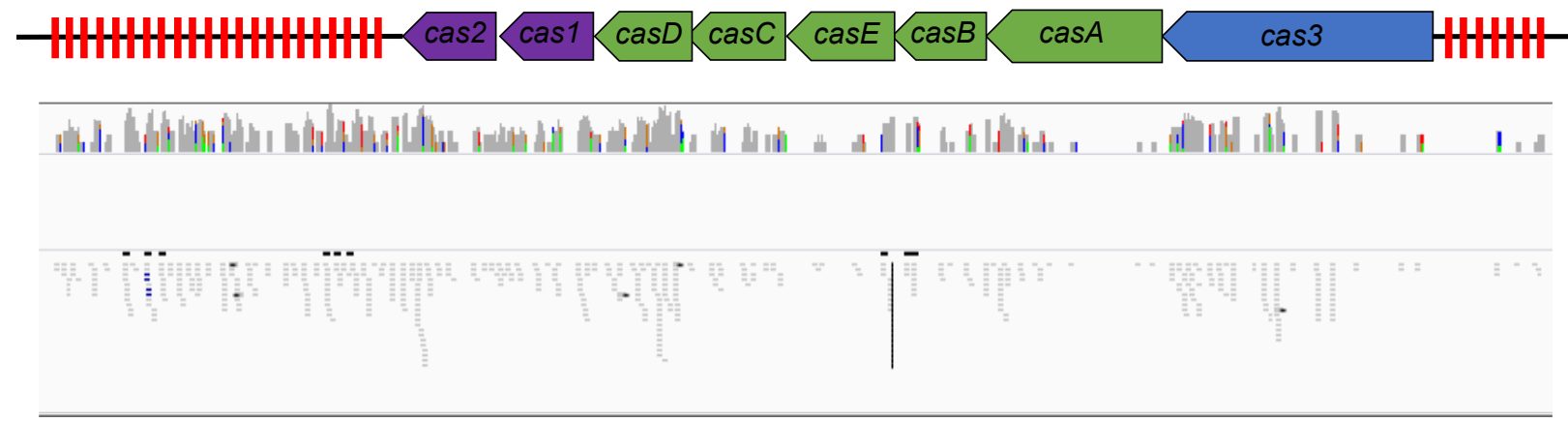

Supplementary Fig. 3 RNA-seq data shows that cas genes and most spacers are expressed in MGH21. 

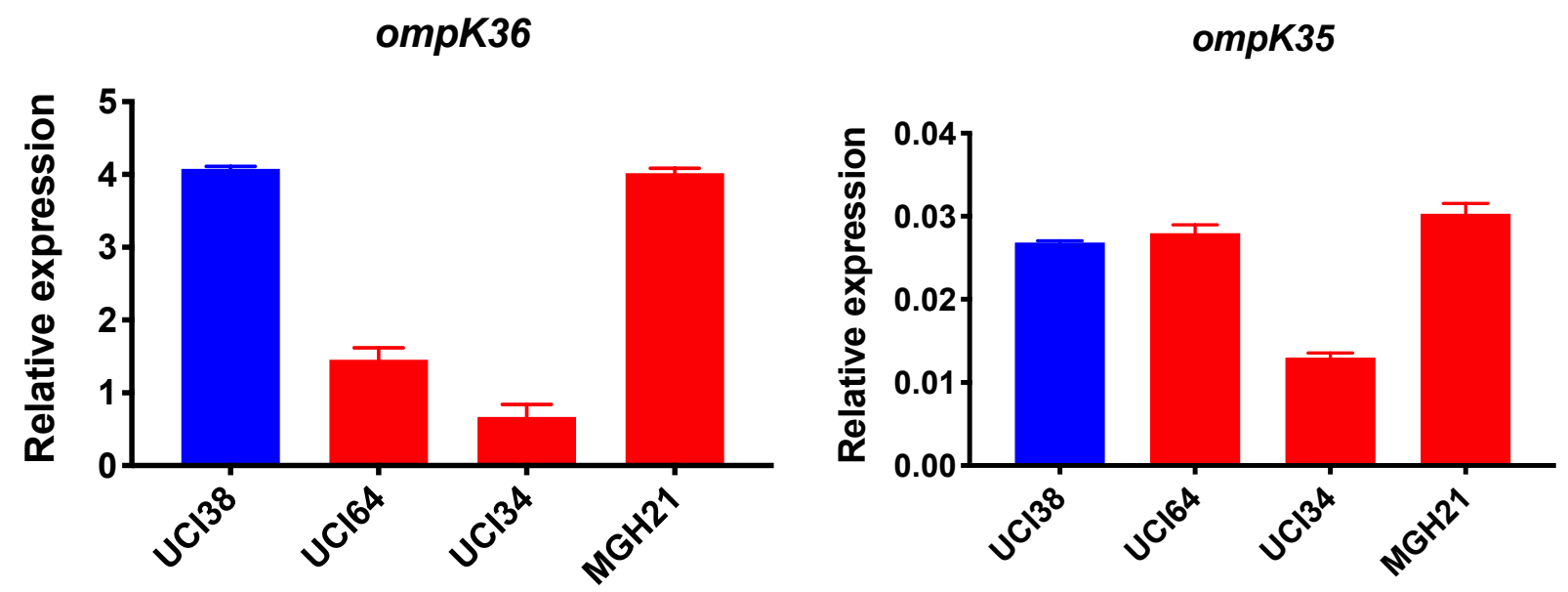

Supplementary Fig.4 Relative expression of ompK36 and ompK35. RT-qPCR data was

normalized to $16 \mathrm{~S}$ rRNA. Experiments were repeated 3 times and error bars were plotted as standard deviation. 


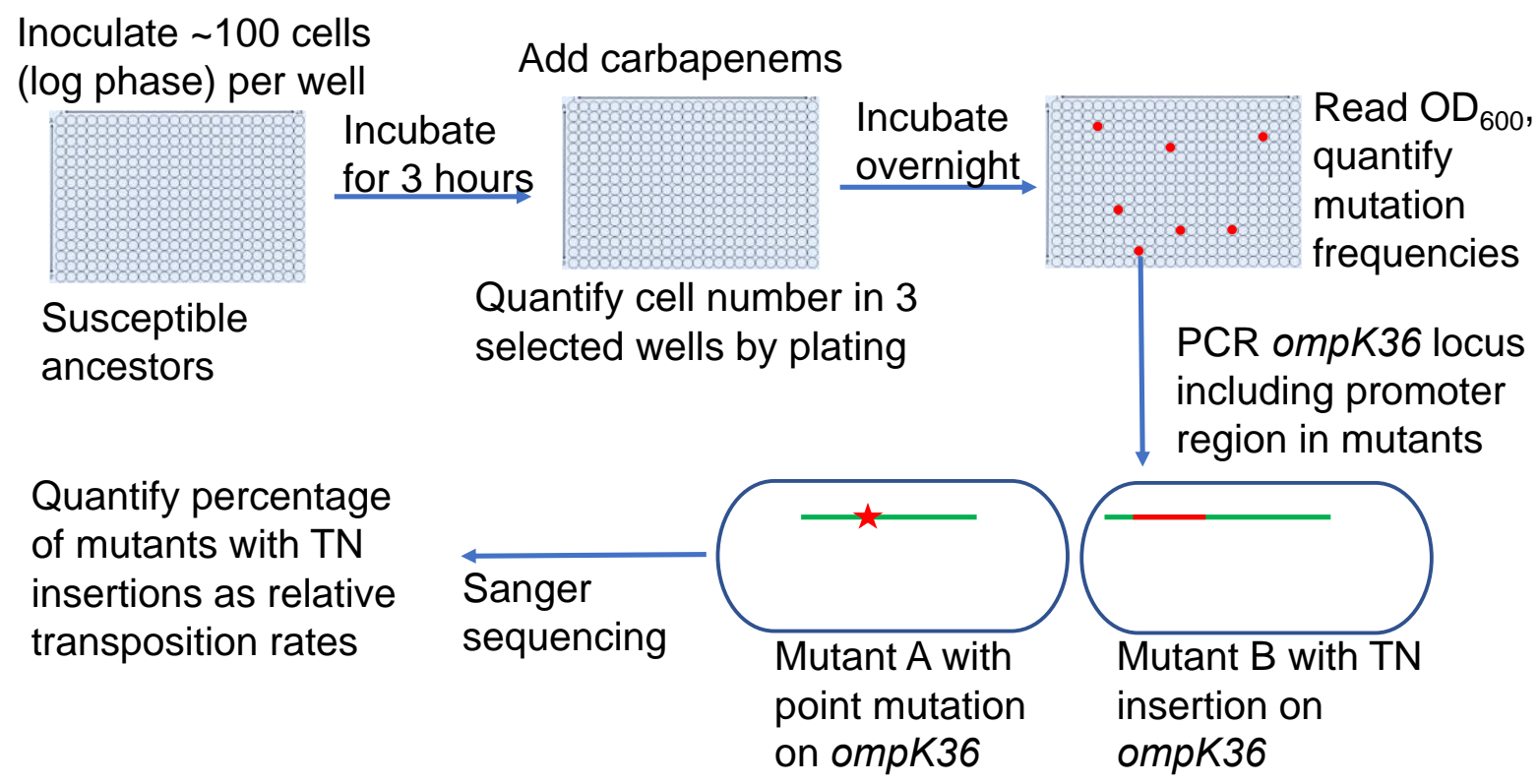

\section{Supplementary Fig. 5 Scheme of the assay for quantification of transposon insertions}

and SNPs in ompK36. Following the isolation of resistant mutants from the modified Luria-

Delbrück system, PCR targeting ompK36 locus, including the upstream 500 bp region, was

performed and the PCR products were Sanger sequenced to determine if there were mutations in the targeted region and the types of mutations. Numbers of mutants carrying transposon insertions or SNPs in ompK36 locus and promoter regions were recorded, and the percentages of mutants with TN insertions or SNP/short INDEL were calculated. 


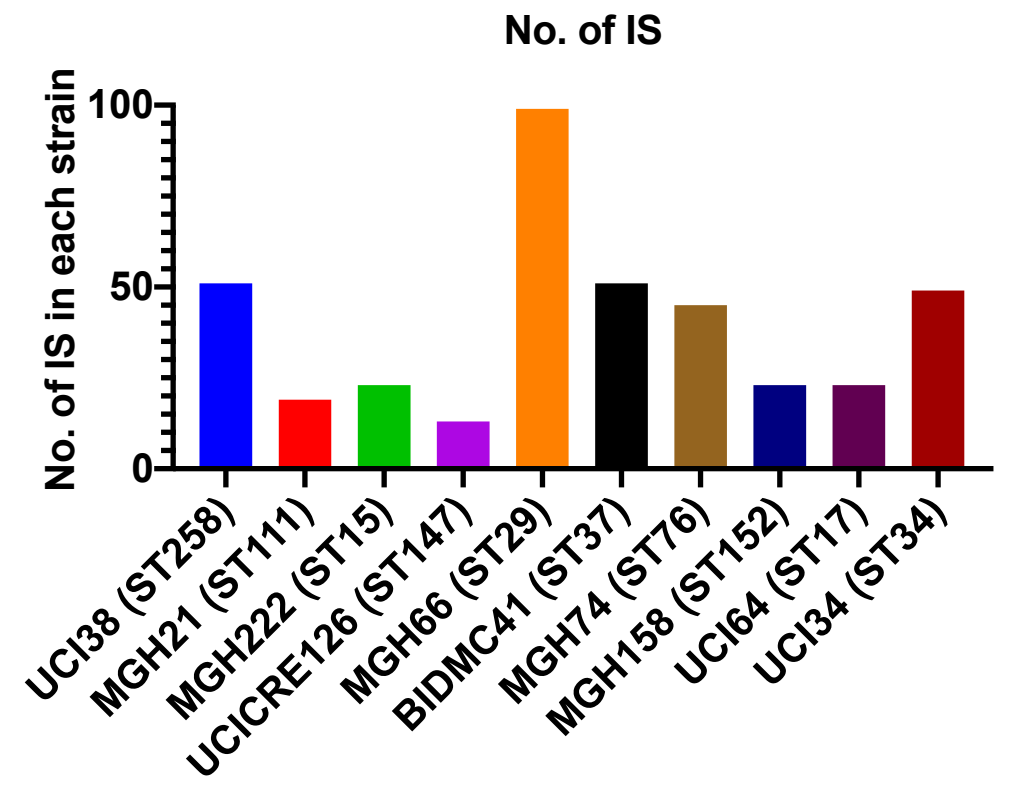

Supplementary Fig. 6 Copy number of ISs in each strain. There is no correlation between the copy number of ISs and the relative level of transposon insertion in ompK36 locus. 


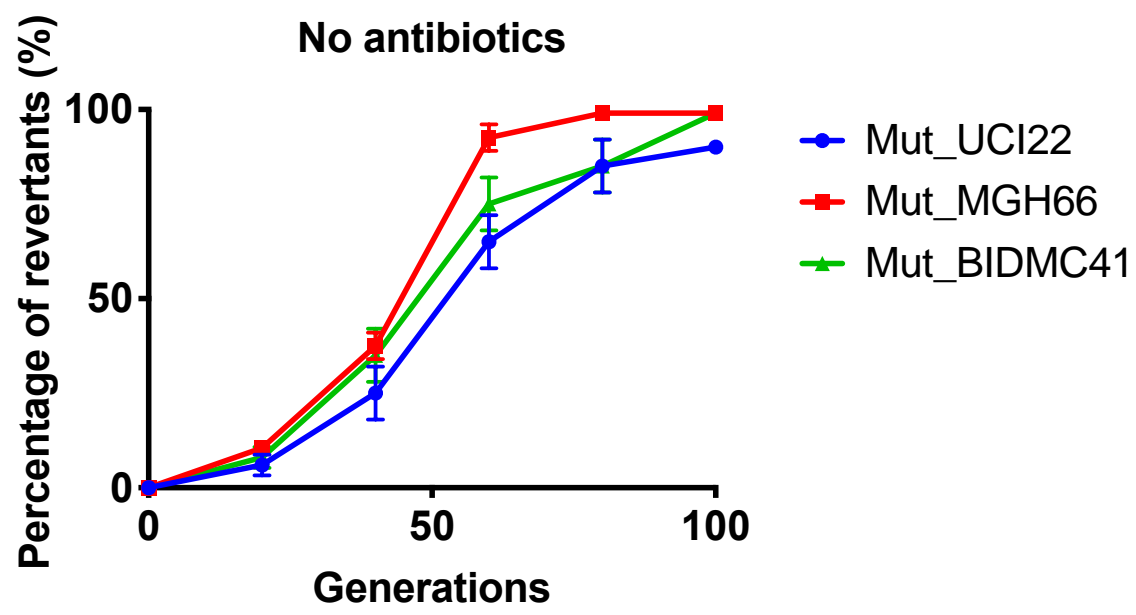

Supplementary Fig. 7 Reversion of TN-insertion mutants derived from UCI22, MGH66 and

BIDMC41. In the absence of antibiotics, mutants carrying transposon insertion in the ompK36 locus and derived from UCI22, MGH66 and BIDMC41 could loss the transposon insertion over $\sim 100$ generations. Experiments were repeated 3 times and error bars were plotted as standard deviation. 

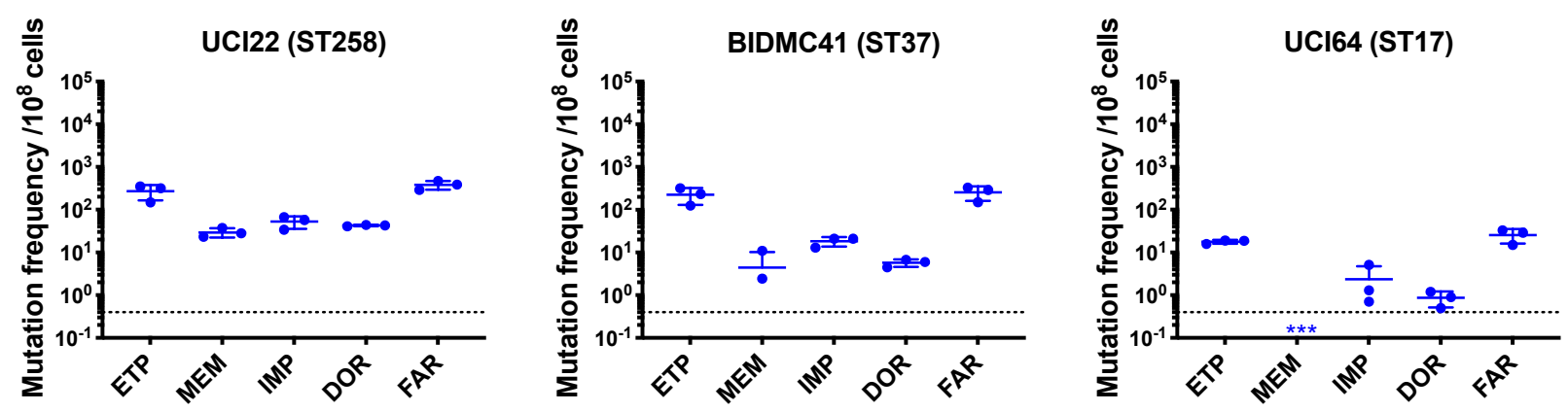

\section{Supplementary Fig. 8 Mutation frequencies of three other isolates under separate} treatment with ertapenem (ETP), meropenem (MEM), imipenem (IMP), doripenem (DOR), or faropenem (FAR). Experiments were repeated 3 times and error bars were plotted as standard deviation. 
a

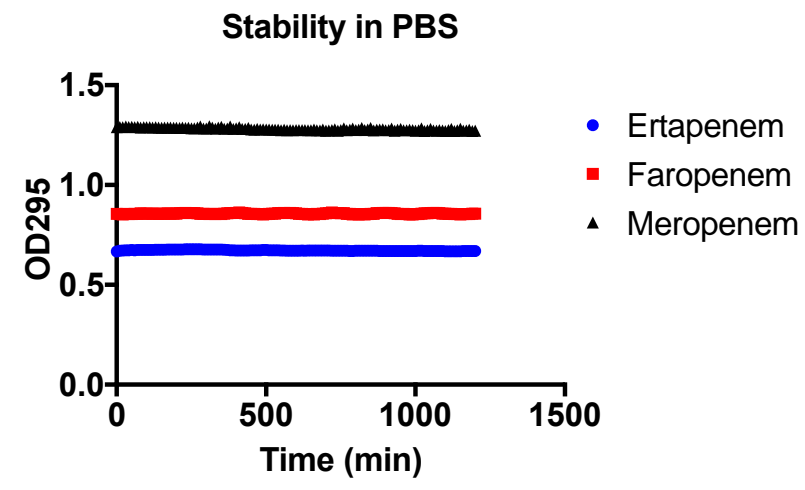

b

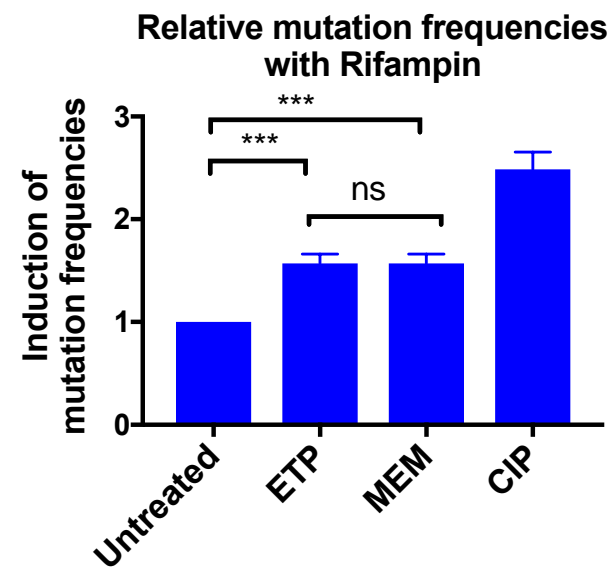

\section{Supplementary Fig. 9 Higher mutation frequencies associated with ertapenem were not}

due to stability of these drugs or to the induction of mutagenesis. a, Stability of ertapenem, faropenem and meropenem in phosphate buffered saline (PBS). Antibiotics were diluted to 0.5 $\mathrm{mM}$ in PBS and $100 \mu \mathrm{l}$ of each antibiotic was used for the assay. $\mathrm{OD}_{295}$ was measured every 10 minutes for 20 hours. These three antibiotics are stable for at least 20 hours in our assay condition. $\mathbf{b}$, Induction of mutation frequencies under treatment with rifampicin. Bacterial cultures of UCI38 in 384-well plates were pre-treated with ertapenem (ETP), meropenem (MEM) or ciprofloxacin (CIP) at $0.1 \times$ MICs of each drug for 2 hours, then mutation frequencies with rifampicin treatment $(50 \mu \mathrm{g} / \mathrm{ml})$ were measured using the modified Luria-Delbrück system. Ertapenem and meropenem induced mutagenesis to the same degree. Data is plotted as the average of three experiments. Error bars are plotted as the standard deviation. Student t-test was used for statistical analysis to compare the untreated cultures with cultures treated with ertapenem or meropenem. 


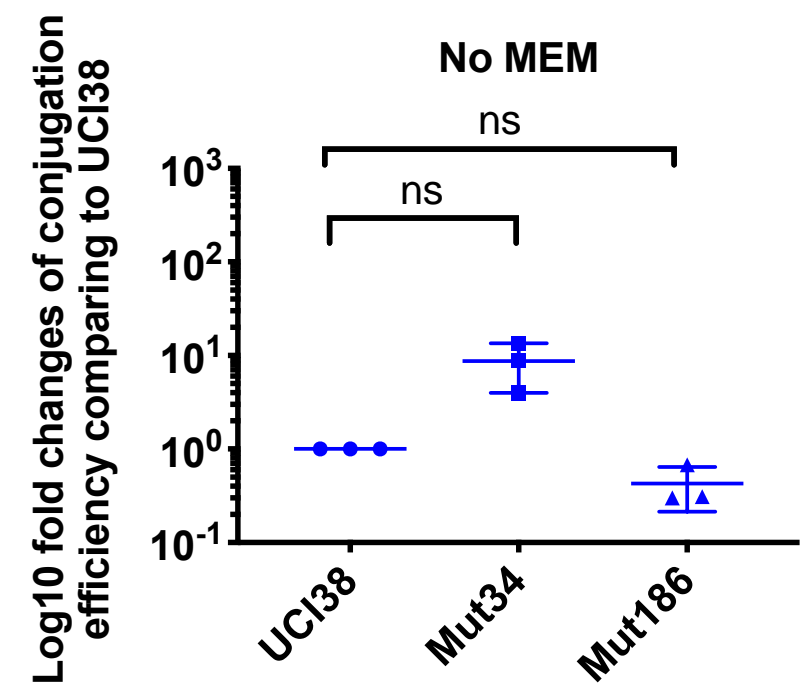

\section{Supplementary Fig. 10 Conjugation efficiencies of UCl38, Mut34 and Mut186 (derived}

from Mut101 with meropenem treatment) with a K. pneumoniae clinical isolate BIDMC45

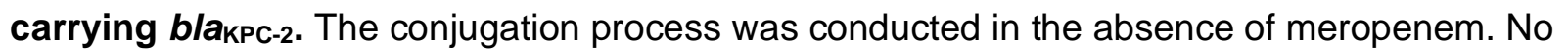
significant difference was observed in the absence of meropenem between UCI38 and Mut34 ( $p$ $=0.11)$ or between $\mathrm{UCl} 38$ and Mut186 $(p=0.16)$. All experiments were repeated three time. Student t-test was used for statistical analysis to compare between the mutant and the ancestor strain UCl38. Error bars are plotted as standard deviation. 
a

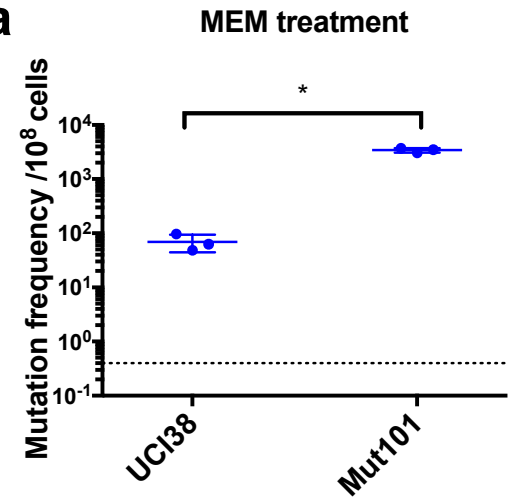

b Conjugation of $b / a_{\mathrm{KPC}-2}$ from a clinical isolate

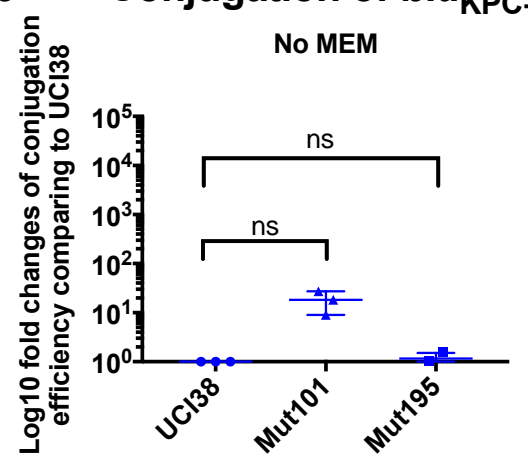

With MEM

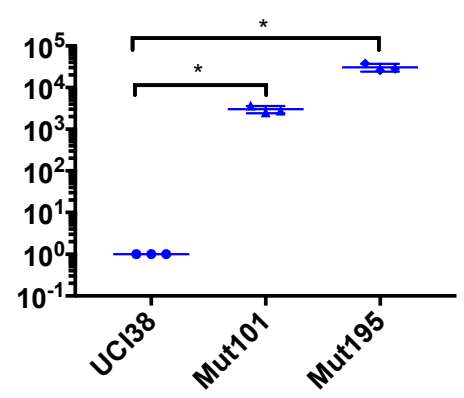

\section{Supplementary Fig. 11 Prior exposure to faropenem promotes the evolution of}

meropenem resistance. a, Mutation frequencies of $\mathrm{UCI} 38$ and Mut101 under treatments with meropenem at the concentration of $1.1 \times \mathrm{MIC}(0.067 \mu \mathrm{g} / \mathrm{ml})$. Mut101 is a mutant of UCI38 derived from faropenem treatment with the same MIC of meropenem as UCI38 and increased MIC of ertapenem. Mut101 showed significantly higher mutation frequencies than these of the UCI38 with meropenem treatment. b, Conjugation efficiencies of UCI38, Mut101and Mut195 (derived from Mut101 with meropenem treatment) with a K. pneumoniae clinical isolate BIDMC45 carrying blakPC-2. The conjugation process was conducted in the absence or presence of meropenem $(0.003 \mu \mathrm{g} / \mathrm{ml})$. In the presence of meropenem, Mut101 and Mut196 showed higher conjugation efficiencies than these of UCI38. No significant difference was observed in the absence of meropenem between UCI38 and Mut101 ( $p=0.08)$ or between UCI38 and Mut195 $(p=0.5)$. All experiments were repeated three time. Student t-test was used for statistical analysis to compare the mutation frequencies (a) or conjugation efficiencies (b) between the mutant and the ancestor strain UCI38. Error bars are plotted as standard deviation. 


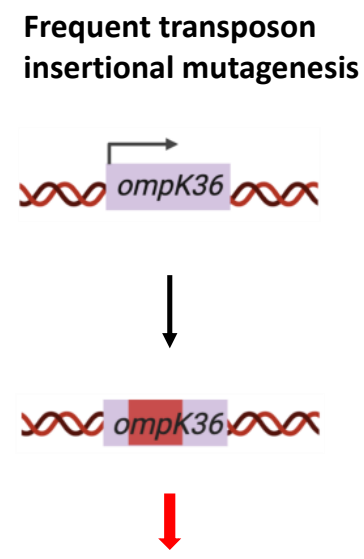

Higher level of spontaneous mutation rates

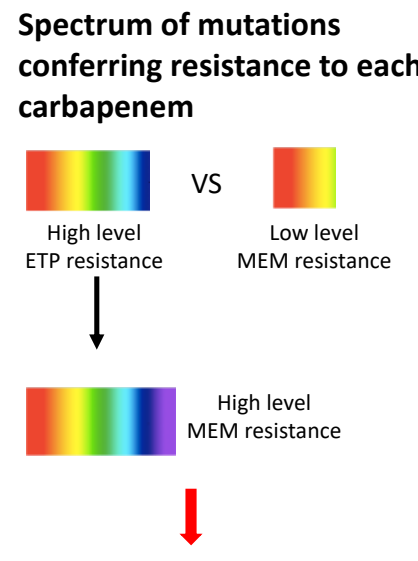

Higher risk of developing resistance to some and/or all carbapenems

\section{Supplementary Fig. 12 Two genetic determinants of the evolution of carbapenem}

resistance were identified from this study. On the one hand, high-level transposon

insertional mutagenesis facilitates the inactivation of porin genes. On the other hand, a broader spectrum of genetic mutation conferring resistance to ertapenem leads to higher rates of developing resistance with ertapenem treatment; these ertapenem-restricted resistance mutations can serve as stepping-stones to facilitate the development of high-level resistance to all carbapenems. 